\title{
Structure and Property of the Singularity Loci of Gough-Stewart Manipulator
}

\author{
Y. $\mathrm{Cao}^{1}, \mathrm{Y} . \mathrm{W} . \mathrm{Li}^{2}$ and Z. Huang ${ }^{2}$ \\ ${ }^{1}$ School of Mechanical Engineering, Jiangnan University, 1800 Lihu Avenue, \\ Wuxi, Jiangsu, 214122, \\ ${ }^{2}$ Robotics Research Center, Yanshan University, Qinhuangdao, Hebei, 066004,
}

China

\section{Introduction}

During the past two decades, parallel manipulator system has become one of the research attentions in robotics. This popularity has been motivated by the fact that parallel manipulators possess some specific advantages over serial manipulators, i.e., higher rigidity and load-carrying capacity, better dynamic performance and a simpler inverse position kinematics, etc. Among various manipulators, the best-known is the Gough-Stewart Platform (GSP) that was introduced as a tire performance (Gough 1956-57) and an aircraft simulator (Stewart 1965).

One of the important problems in robot kinematics is special configuration or singularity. As to parallel manipulators, in such configurations, the end-effector keeps at least one remnant freedom while all the actuators are locked. This transitorily puts the end-effector out of control. Meanwhile, the articular forces may go to infinity and cause mechanical damages.

Determination of the special configurations of the six-DOF Gough-Stewart parallel manipulators is a very important problem. It is one of the main concerns in the analysis and design of manipulators. The singularity analysis of parallel manipulators has attracted a great deal of attention in the past two decades. Hunt (1983) first discovered a special configuration for this manipulator that occurs when the moving triangle-platform is coplanar with two legs meeting at a vertex of the triangle, and all the six segments associated with six prismatic actuators intersect a common line. Fichter (1986) discovered a singularity of the parallel manipulator. That occurs when the moving platform rotates $\psi$ $= \pm \pi / 2$ around Z-axis, whatever the position of the moving platform is. That mechanism has a triangular mobile platform and a hexagonal base platform. It may be named a 3/6-GSP. Huang and Qu (1987) and Huang, Kong and Fang (1997) also studied the singularity of the parallel manipulator, whose moving and basic platforms are both semi-regular hexagons (6/6-GSP). It also occurs when $\psi= \pm \pi / 2$. Merlet $(1988,1989)$ studied the singularity of the six-DOF 3/6-GSP more systematically based on Grassman line geometry. He discovered many new singularities including $3 c, 4 b, 4 d, 5 a$ and $5 b$. $3 c$ occurs when four lines of the six legs intersect at a common point; $4 \mathrm{~b}$ occurs when five lines are concurrent with two skew lines; $4 \mathrm{~d}$ occurs when all the five lines are in one plane or pass through one common point 
in that plane; $5 \mathrm{a}$ is in general complex; $5 \mathrm{~b}$ occurs when the six segments cross the same line. Based on line geometry, wrench singularity analyses for platform devices have been presented by Collins \& Long (1995), and Hao \& McCarthy (1998). Gosselin and Angeles (1990) pointed that singularities of closed-loop mechanisms can be classified into three different groups based on the Jacobian matrices. This classification was further discussed by Zlatanov, Fenton and Benhabib (1994, 1995). Zlatanov, Bonev and Gosselin (2002) discussed constraint singularities. Ma and Angeles (1991) studied architecture singularities of parallel manipulators. Kong (1998) also discussed architecture singularities of the general GSP. McAree and Daniel (1999) discussed the singularity and motion property of a 3/3-parallel manipulator. Karger and Husty (1998), Karger (2001) described the singular positions and self-motions of a special class of planar parallel manipulators where the platform is similar to the base one. It is shown that it has no self-motions unless it is architecturally singular. Kong (1998), Kong and Gosselin (2002) also studied self-motion. Chan and Ebert-Uphoff (2000) studied the nature of the kinematic deficiency in a singular configuration by calculating the nullspace of the Jacobian matrix. Di Gregorio (2004) studied the SX-YS-ZS Structures and Singularity.

Many researches dealt only with isolated singular points in space. However, in the practical configuration space of parallel manipulators the singularity configuration should be a continuous singularity curve or even be high-dimension surface. One of the main concerns is further to find out its singularity loci and their graphical representations, as well as the structure and property of the singularity loci. That is of great significance in a context of analysis and design since it allows one to obtain a complete picture of the location of the singular configurations in the workspace. For a given practical application, it is therefore easy to decide whether the singularities can be avoided. Sefrioui and Gosselin (1994, 1995) studied singularity loci of planar and spherical parallel mechanisms. Wang and Gosselin $(1996,1997)$ used the numerical method to study the singularity loci of spatial four- and fiveDOF parallel manipulators. Collins and McCarthy $(1997,1998)$ studied singularity loci of the planar 3-RPR parallel manipulator, and 2-2-2 and 3-2-1 platforms and obtained cubic singularity surfaces. For the six-DOF Gough-Stewart Platform, however, the singularity expression generally is quite complicated, and difficult to analyze. Recently, Wang (1998) presented a method to analyze the singularity of a special form of the GSP and derived corresponding analytical singularity conditions. Di Gregorio $(2001,2002)$ also discussed the singularity loci of 3/6 and 6/6 fully-parallel manipulators. In particular, Mayer St-Onge and Gosselin (2000) analyzed the Jacobian matrix of general Stewart manipulators by two different new approaches. They derived a simpler explicit expression from the Jacobian matrix, and pointed out that the singularity locus of the general Gough-Stewart manipulator should be a polynomial expression of degree three. They also gave the graphical representations of the singularity loci.

For practical application, we want to obtain a simpler algebra expression of the singularity loci, their accurate graphical representations and know whether it consists of some typical geometrical figures. But this is very difficult for the Gough-Stewart manipulator. Huang et al. $(1999,2003)$ studied the singularity kinematics principle of parallel manipulators, and proved a new kinematics sufficient and necessary condition to determine the singularity. Using this method he discovered the characters of singularity locus of the 3/6-GoughStewart platform firstly. It shows that the singularity locus of the 3/6-Gough-Stewart platform is resolvable and consists of two typical geometrical graphs, a plane and a 
hyperbolic paraboloid, for the special orientations: $\phi= \pm 30^{\circ}, \pm 90^{\circ}$, or $\pm 150^{\circ}$. However, the singularity locus expression of degree three is irresolvable, and the locus graph in infinite parallel principal sections includes a parabola, four pairs of intersecting straight lines and infinite hyperbolas for the general orientations: $\phi \neq \pm 30^{\circ}, \pm 90^{\circ}$, and $\pm 150^{\circ}$.

For the singularity loci of the 6/6-GSP which is a more general structure form and widely used in practice, its graphical representations of the singularity loci for different orientations are quite various and complex. Huang and Cao (2005) analyzed the singularity loci both in 3-D space and in the principal-section on which the moving platform lies. The singularity locus equation of this class of Gough-Stewart manipulators in three-dimensional space is also irresolvable, and the curves in infinite parallel principal sections of the singularity loci also contains one parabola, four pairs of intersecting straight lines, and infinite hyperbolas. We also found out an incredible phenomenon, in that special configuration six lines associated with the six extensible links of the 6/6-Gough-Stewart manipulator can intersect the same common line and the remnant instantaneous motion of the manipulator is a pure rotation.

All the above-mentioned analyses are only about positional singularity when the orientation of the moving platform is specified and invariable. On the other hand, there is a need to further find out the orientation-singularity space when the position of the moving platform is specified and invariable. Some researchers began to study the issue, such as Pernkopf and Husty (2002); Cao, Huang \& Ge (2006). Of course, for this topic there is still much work to be done in depth.

\section{The kinematics principle and linear-complex classification}

\subsection{The classification of singularity by linear-complex}

A general algebraic equation for a linear complex (Hunt 1978; Ball 1900) is:

$$
a_{1} P+a_{2} Q+a_{3} R+a_{4} L+a_{5} M+a_{6} N=0
$$

where the six coefficients denote a twist screw

$$
\mathbf{\$}^{m}=\left(a_{1} a_{2} a_{3} ; a_{4} a_{5} a_{6}\right)
$$

Its pitch is

$$
h^{m}=\frac{a_{1} a_{4}+a_{2} a_{5}+a_{3} a_{6}}{a_{1}^{2}+a_{2}^{2}+a_{3}^{2}}
$$

Its reciprocal screw satisfying Eq. (1) is

$$
\$=\left(\begin{array}{lllll}
L & M & N
\end{array} \quad Q \quad R\right)
$$

and we have

$$
L P+M Q+N R=0
$$

where $\$$ denotes a line vector. The infinite line vectors satisfying Eq. (1) composed a line complex. 


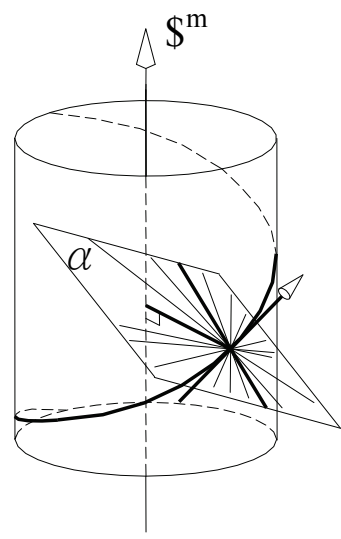

Fig. 1. Linear complex

In a linear complex (Hunt 1978), those lines that pass through any pole must all lie in the same polar plane; those lines that lie in any polar plane must all intersect the same point. Fig. 1 shows the pole and polar plane of a linear complex with the pencil of lines in $\alpha$. All the lines that pass through the pole are normal to the helix. The linear complex can be divided into three parts according to its pitch $h^{m}$ : when $h^{m}$ is finite and nonzero, it is a general linear complex; when $h^{m=0}$, this is the first special linear complex, in which all the coaxial helices collapse into homocentric circles with a common axis $\$^{m}$ and all the lines of the complex intersect $\$^{m}$ or parallel to it; and when $h^{m=\infty}$, this is the second special linear complex, in which all the lines of the complex comprise planar fields of lines in all planes normal to the direction $\$^{m}$, and $\$^{m}$ is no longer occupying a specific line. The last two forms are associated respectively with pure rotation and pure translation.

All singularities of the Gough-Stewart parallel mechanism belong to the linear-complex singularity. From this point of view, the singularity can be divided into three kinds with different instant output motion:

(1) The general Linear-Complex Singularity. The possible motion of end-effector is a twist with $h^{m}$ is finite and nonzero;

(2) The First Special Linear-Complex Singularity. The possible motion of end-effector is a pure rotation with $h^{m=0}$;

(3) The Second Special Linear-Complex Singularity. The possible motion of end-effector is a pure translation with $h^{m=\infty}$.

\subsection{The kinematic principle of singularity}

First of all, let us discuss the velocity relationship of three points in a moving body. The following issue is to introduce the principle of a novel method analyzing the singularities of parallel manipulators (Huang et al. 1999; 2003; Ebert-Uphoff et al., 2000; Kong and Gosselin 2001). Let us consider any non-collinear three points in a rigid body, and then we may deduce the following theorem:

Theorem 1: Three velocities of three points in a moving body have three normal planes at the corresponding three points. In general, the three planes intersect at a common point, and the intersecting point necessarily lies in the plane determined by the three points. 


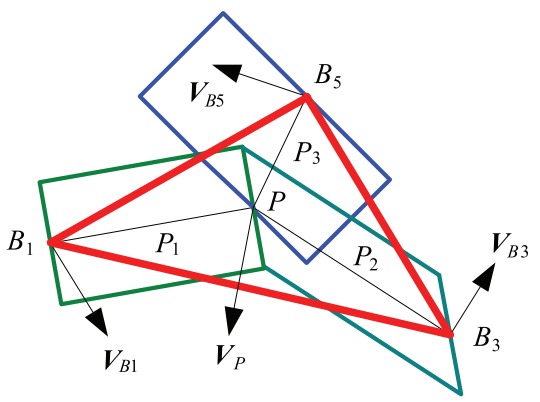

Fig. 2 The velocity relationship of three non collinear points in a moving body

Theorem 2: When three velocity directions of three points in a rigid body are given, then three normal planes of the three velocities are determined. If the intersecting point of the three planes lies in the plane determined by the three points, the three velocities can determine a twist; otherwise, the given velocities are improper and cannot determine a twist of that body.

The thinking of the velocity analysis in the proof of Theorem 2 itself is also useful for singularity study of the 3/6-GSP.

The 3/6-GSP is a typical manipulator which many authors paid attention to. The 3/6-GSP is represented schematically in Fig.3. It consists of a mobile platform $B_{1} B_{3} B_{5}$, equilateral triangle; a base platform $C_{1} \ldots C_{6}$, semi-regular hexagon; and they are connected via six extensible prismatic actuators.

When all the legs of 3/6-GSP are locked, the three normal planes of three velocities $V_{B 1}, V_{B 3}$ and $V_{B 5}$ are respectively $B_{1} C_{1} C_{2}, B_{3} C_{3} C_{4}$ and $B_{5} C_{5} C_{6}$ (Fig.3). According to Theorem 2, we may educe the following deduction to determine the singularity of 3/6-GSP. Let us firstly define a "Star-frame $C-B_{1} B_{3} B_{5}$ " in the moving platform. It is constructed by using three ray lines passing three points, $B_{1}, B_{2}$ and $B_{3}$, of the triangle $B_{1} B_{3} B_{5}$ and intersecting at a common point $C$ called the center of Star-Frame.

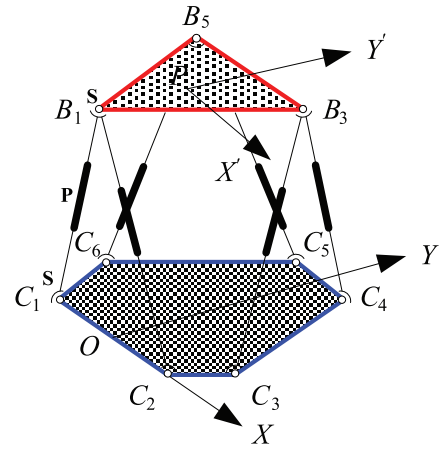

a) A 3/6-Stewart manipulator

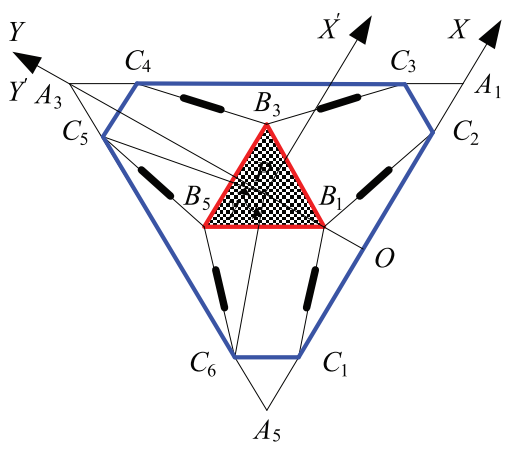

b) Its top view

Fig. 3. A 3/6-Stewart parallel manipulator 
Theorem 3: A necessary and sufficient condition that the three velocities of three points in a rigid body can express that the body has a possible twist motion is that the intersecting point of three normal planes of the three velocities lies in the plane determined by the three points.

\section{Structure and property of singularity loci of 3/6-Gough-Stewart for special orientations}

The kinematics method can determine the singularity of the manipulator. If the six extensible legs of the GSP are locked and the mechanism has an instantaneous freedom, the manipulator is singular. Now, let us firstly discuss the kinematics properties of the typical singularity structures including singularities: 3c, 4b, 4d, 5b (Merlet 1988; 1989) and others.

\subsection{Singularity hyperbola equation derived in an oblique plane}

Our task is to find the whole singularity loci of the GSP and identify their structure and property. It is of an important and difficult issue. Here three Euler angles $\phi, \theta$ and $\psi$ are used to represent orientation of the mobile in terms of a rotation $\phi$ about $Z$-axis, then a rotation $\theta$ about the new $Y^{\prime}$-axis, and finally a rotation $\psi$ about the new $Z$ "-axis.

In order to find the whole singularity loci and solve the issue, we first study the singularity equation in a special plane (Huang et al. 2003). The issue is divided into two parts:

(1) When the first Euler angle $\phi$ is equal to one of the following values, $\pm 30^{\circ}, \pm 90^{\circ}$, and $\pm 150^{\circ}$, it is a special orientation cases and easier to analyze.

(2) When $\phi$ is any value with the exception of $\pm 30^{\circ}, \pm 90^{\circ}$, and $\pm 150^{\circ}$, this is the general case.

Now, we solve the equation for singularity curve of the 3/6-GSP in a certain plane while the orientation of the mobile is provisionally set to $\phi=90^{\circ}, \psi=0$ and $\theta$ is any finite nonzero value.

The parameters of the parallel manipulator are as follows. The circumcircle radius of the basic hexagon platform is $R_{a}$, and the one of the triangle mobile is $R_{b} ; \beta_{0}$ denotes the central angle of the circumcircle of the basic hexagon corresponding to side $C_{1} C_{2}$. Point $P$ is the geometric center of the mobile (Fig. 3). The stationary frame $O-X Y Z$ is fixed to the base and the moving frame $P-X^{\prime} Y^{\prime} Z^{\prime}$ is attached to the mobile.

Fig. 4 shows the position after the mobile rotates $\left(90^{\circ}, \theta, 0\right)$. The oblique plane in which the moving platform lies intersects the basic plane at line $U V$, which is parallel to axis $X$. For the orientation, $B_{1} P\left(Y^{\prime}\right)$ is parallel to $A_{5} A_{1}(\mathrm{X})$.At first, providing that point $P$ is located at a special point $C_{0}$ in the perpendicular bisector of $U V$, and the distance from $O_{2}$ to point $C_{0}$ is equal to that between point $O_{2}$ and $A_{3}$, then we deduce that $C_{0} B_{3}$ and $A_{3} A_{1}$ intersect at point $V$, and $C_{0} B_{5}$ and $A_{3} A_{5}$ intersect at $U$. In that case, the mechanism is singular according to Deduction 2. The included angle between the oblique plane and the basic one is $\theta$. In order to conveniently express the oblique plane below, we call it $\theta$-plane. Let us suppose that the mobile translates to the position $B_{11} B_{31} B_{51}$ in $\theta$-plane and line $B_{11} P$ intersects line $O_{2} C_{0}$ at $C$. If line $B_{31} C$ intersects $A_{1} A_{3}$ at point $V$, and line $C_{1} B_{51}$ intersects $A_{3} A_{5}$ at point $U$, the mechanism is also singular (Deduction 2). We can prove that the center of Star-frame always lies in line $\mathrm{O}_{2} \mathrm{C}_{0}$ for the orientation. In general, the singularity is a general-linear-complex singularity. Based on the analysis above, we study the singularities of 3/6-GSP when the mobile translates arbitrarily in $\theta$-plane. The coordinates of point $\mathrm{C}_{0}$ and $\mathrm{O}_{2}$ with respect to the fixed frame are $\left(0, Y_{0}, Z_{0}\right)$ and $(0, u, 0)$, respectively. The frame $O_{2}-x y z$ is attached to $\theta$-plane. It should be noticed that angle $\theta$ as shown in Fig. 5 about the $Y^{\prime}$-axis is negative. 


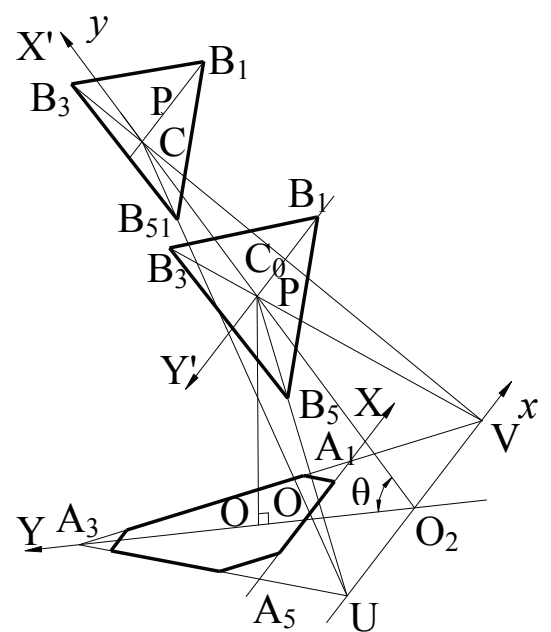

Fig. 4. $\theta$-Oblique plane for the orientation $\left(90^{\circ}, \theta, 0\right)$

The coordinates of points $P, C, B_{31}$ and $V$ with respect to $O_{2}-x y z$ are

$$
\begin{aligned}
& P:(x, y, 0) \\
& C:(0, y, 0) \\
& B_{31}:\left(x-\frac{R_{b}}{2}, y+\frac{\sqrt{3}}{2} R_{b}, 0\right) \\
& V:\left(-\frac{\sqrt{3}}{3} \frac{Z_{0}}{\sin \theta}, 0,0\right)
\end{aligned}
$$

Considering $\mathrm{O}_{2} \mathrm{C}_{0}=\mathrm{O}_{2} \mathrm{~A}_{3}$, we can obtain

$$
\mathrm{O}_{2} \mathrm{O}_{1}-O \mathrm{O}_{1}+O A_{3}=\mathrm{O}_{2} \mathrm{C}_{0}
$$

namely

$$
3 R_{a} \cos \left(\beta_{0} / 2\right)-Z_{0} \frac{\cos \theta}{\sin \theta}-Y_{0}=-\frac{Z_{0}}{\sin \theta}
$$

In the right-angled triangle $\Delta \mathrm{O}_{1} \mathrm{O}_{2} \mathrm{C}_{0}$, we obtain

$$
Y_{0}-u=-Z_{0} \frac{\cos \theta}{\sin \theta}
$$

Solving Eqs. (8) and (9) for $Y_{0}$ and $Z_{0}$, we obtain

$$
\begin{aligned}
& Y_{0}=u(1-\cos \theta)+3 R_{a} \cos \left(\beta_{0} / 2\right) \cos \theta \\
& Z_{0}=u \sin \theta-3 R_{a} \cos \left(\beta_{0} / 2\right) \sin \theta
\end{aligned}
$$

Provided that the coordinates of an arbitrary point in line $B_{31} \operatorname{Vare}\left(x_{x}, y_{y}, 0\right)$, its equation is written as 


$$
\frac{y_{y}-y-\frac{\sqrt{3}}{2} R_{b}}{-y-\frac{\sqrt{3}}{2} R_{b}}=\frac{x_{x}-x+\frac{R_{b}}{2}}{-\frac{\sqrt{3}}{3} \frac{Z_{0}}{\sin \theta}-x+\frac{R_{b}}{2}}
$$

Since point $C$ lies in line $B_{31} V$, substitute the coordinates of point $C\left(x_{x}=0\right.$ and $\left.y_{y}=y\right)$ into Eq. (9) and simplify as

$$
x y-\frac{R_{b}}{2} y-\frac{Z_{0} R_{b}}{2 \sin \theta}=0
$$

Substituting Eq. (10) into Eq. (11) and eliminating $Z_{0}$, yield

$$
x y-\frac{R_{b}}{2} y+\frac{\left(3 R_{a} \cos \left(\beta_{0} / 2\right)-u\right) R_{b}}{2}=0
$$

Eq. (13) denotes a hyperbola and is independent of the Euler angle $\theta$. The coordinates of its center are $\left(R_{b} / 2,0\right)$, and its vertical and horizontal asymptotes are $x=R_{b} / 2, y=0$

This is an important conclusion, as we have known that the singularity equation of GSP in 3dimension space is a polynomial expression of degree three. However, equation (13) is only a quadratic equation in the special $\theta$-plane. Eq. (13) only contains variables $x$ and $y$, so it denotes the positions of point $p$ when the mechanism is singular. The equation is termed the equation of the singularity curve in $\theta$-plane.

When orientation of the mobile is given by three Euler angles $\left(-90^{\circ}, \theta, 0\right)$, the singularity equation can also be obtained in $\theta$-plane with respect to the frame $\mathrm{O}_{2}-x y z$, the same as in Fig. 4 .

$$
x y+\frac{R_{b}}{2} y-\frac{\left(3 R_{a} \cos \left(\beta_{0} / 2\right)-u\right) R_{b}}{2}=0
$$

When parameters of the mechanism are set to $R_{a}=\sqrt{2}, R_{b}=1, \beta_{0}=90^{\circ}$ and $u=-2$, the hyperbolas denoted by Eqs. (13) and (14) are illustrated in Fig. 6(a). Since the result comes from the above-mentioned Theorem and satisfies the necessary and sufficient condition of singularity, so that there is no any singularity except the points on hyperbolas in that $\theta$-plane.

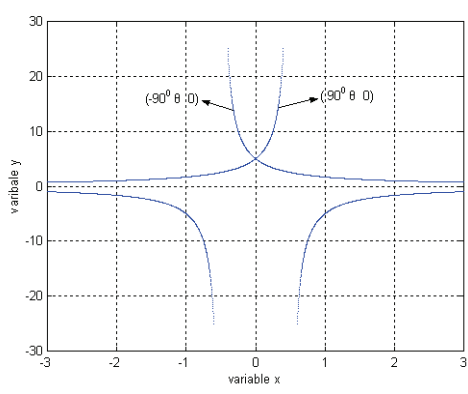

(a) For the orientation $\left( \pm 90^{0} \theta\right.$ 0)

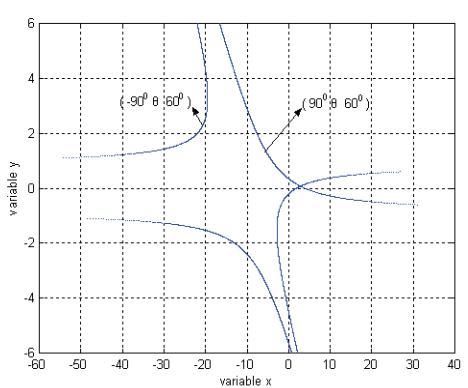

(b) For the orientation $\left( \pm 90^{0} \theta 60^{0}\right)$

Fig. 5. The singularity curve in $\theta$ - plane 


\subsection{The singularity equation derived in three-dimensional space}

Eqs. (13) and (14) are deduced by geometric method in the oblique plane. By Theorem 3, we can analyze the distribution properties of the singularities of 3/6-GSP in three-dimensional space.

The coordinates of point $B_{i}(i=1,2,3)$ of the mobile are denoted as $B_{i}{ }^{\prime}:\left(B_{i x}{ }^{\prime}, B_{i y^{\prime}}{ }^{\prime}, B_{i z}{ }^{\prime}\right)$ in the moving frame, and $\boldsymbol{B}_{i}:\left(B_{i x}, B_{i y}, B_{i z}\right)$ in the fixed frame; the coordinates of point $\boldsymbol{C}_{j}$ are denoted as $\left(C_{j x}, C_{j y}, C_{j z}\right)$ in the fixed frame.

The transformation matrix $\mathbf{T}$ of the moving frame with respect to the fixed one can be written using Euler angles $\phi, \theta$ and $\psi$ as

$$
[\mathbf{T}]=\left[\begin{array}{llll}
\cos \phi \operatorname{os} \theta \cos \psi-\sin \phi \sin \psi & -\cos \phi \cos \theta \sin \psi-\sin \phi \cos \psi & \cos \phi \sin \theta & X \\
\sin \phi \cos \theta \cos \psi+\cos \phi \sin \psi & -\sin \phi \cos \theta \sin \psi+\cos \phi \cos \psi & \sin \phi \sin \theta & Y \\
-\sin \theta \cos \psi & \sin \theta \sin \psi & \cos \theta & Z \\
0 & 0 & 0 & 1
\end{array}\right]
$$

where $(X, Y, Z)$ are the coordinates of point $p$ with respect to the fixed frame. The coordinates of point $B_{i}$ in the mobile with respect to the fixed frame are

$$
\left\{\begin{array}{l}
B_{i x} \\
B_{i y} \\
B_{i z} \\
1
\end{array}\right\}=[\mathbf{T}]\left\{\begin{array}{l}
B_{i x}^{\prime} \\
B_{i y}^{\prime} \\
B_{i z}^{\prime} \\
1
\end{array}\right\}, \quad i=1,2,3
$$

\subsubsection{Singularity equation for orientation $\left(90^{\circ}, \theta, 0\right)$}

When three Euler angles are $90^{\circ}, \theta$ and 0, respectively, from Eq. (16), we can obtain coordinates of three points $B_{i}(i=1,2,3)$ in the mobile with respect to the fixed frame. Thus three equations of three normal planes $B_{1} C_{1} C_{2}, B_{3} C_{3} C_{4}$ and $B_{5} C_{5} C_{6}$ and the one that the mobile belongs to can be written by the coordinates of the three corresponding points. The equation of plane $B_{1} C_{1} C_{2}$ is

$$
\left|\begin{array}{lll}
x-B_{1 x} & y-B_{1 y} & z-B_{1 z} \\
C_{1 x}-B_{1 x} & C_{1 y}-B_{1 y} & C_{1 z}-B_{1 z} \\
C_{2 x}-B_{1 x} & C_{2 y}-B_{1 y} & C_{2 z}-B_{1 z}
\end{array}\right|=0
$$

where $x, y$ and $z$ are the coordinates of moving point in plane $B_{1} C_{1} C_{2}$ with respect to the fixed frame. Substituting coordinates of points $B_{1}, C_{1}$ and $C_{2}$ into the above equation, we obtain

$$
Z y-Y z=0
$$

Similarly, the equation of plane $B_{3} C_{3} C_{4}$ can be obtained

$$
\begin{aligned}
& \left(-3 R_{b} \sin \theta+2 \sqrt{3} Z\right) x+\left(2 Z-\sqrt{3} R_{b} \sin \theta\right) y+\left(-2 Y+6 R_{a} \cos \left(\beta_{0} / 2\right)-\sqrt{3} R_{b} \cos \theta\right. \\
& \left.+\sqrt{3} R_{b}-2 \sqrt{3} X\right) z-6 Z R_{a} \cos \left(\beta_{0} / 2\right)+3 \sqrt{3} R_{a} R_{b} \sin \theta \cos \left(\beta_{0} / 2\right)=0
\end{aligned}
$$


The one of plane $B_{5} C_{5} C_{6}$ is:

$$
\begin{aligned}
& \left(-3 R_{b} \sin \theta-2 \sqrt{3} Z\right) x+\left(2 Z+\sqrt{3} R_{b} \sin \theta\right) y+\left(-2 Y+6 R_{a} \cos \left(\beta_{0} / 2\right)+\sqrt{3} R_{b} \cos \theta-\sqrt{3} R_{b}\right. \\
& +2 \sqrt{3} X) z-6 Z R_{a} \cos \left(\beta_{0} / 2\right)-3 \sqrt{3} R_{a} R_{b} \sin \theta \cos \left(\beta_{0} / 2\right)=0
\end{aligned}
$$

The one of plane $B_{1} B_{3} B_{5}$ is

$$
(\sin \theta) \mathrm{y}+(\cos \theta) z-(\sin \theta) Y-(\cos \theta) \mathrm{Z}=0
$$

Note that, the equations of these planes are on the same condition that point $P(X, Y, Z)$ is located at some point and the orientation is denoted by three Euler angles $\left(90^{\circ}, \theta, 0\right)$.

Solving Eqs. (18), (19) and (20) for $x, y$ and $z$, then substituting them into Eq. (21) and eliminating $x, y$ and $z$, we obtain

$$
[(\sin \theta) Y+(\cos \theta) Z]\left[2 X Z+R_{b}(\sin \theta) Y+R_{b}(\cos \theta) Z-R_{b} Z-3 R_{b} R_{a} \sin \theta \cos \left(\beta_{0} / 2\right)\right]=0
$$

According to Theorem 3, Eq. (22) denotes the singularity locus of point $P$ for the orientation $\left(90^{\circ}, \theta, 0\right)$. Obviously, it includes a plane and a conicoid. The plane equation is

$$
(\sin \theta) Y+(\cos \theta) Z=0
$$

Eq. (23) denotes that singularity locus of point $P$ is a plane containing line $C_{1} C_{2}$ or $A_{5} A_{1}$, namely, $X$-axis. As the plane and plane $B_{1} B_{3} B_{5}$ denoted by Eq. (23) have the same normal vector, and when plane $B_{1} B_{3} B_{5}$ translates and coincides with plane expressed by Eq.(23), the configuration is singular. The case belongs to the Hunt's singularity and is the first speciallinear-complex singularity explained in Case 5. Eq. (23) shows that the mechanism is singular, wherever point $P$ locates in the plane.

The conicoid equation is

$$
2 X Z+R_{b}(\sin \theta) Y+R_{b}((\cos \theta)-1) Z-3 R_{b} R_{a} \sin \theta \cos \left(\beta_{0} / 2\right)=0
$$

When $\theta$ is constant, Eq. (24) denotes a hyperbolic paraboloid and we will explain later. Eq. (28) also represents a hyperbolic paraboloid.

\subsubsection{Singularity equation for the orientation $\left( \pm 90^{\circ}, \theta, \psi\right)$}

\subsubsection{The Derivation of the Equation}

For the orientation $\left( \pm 90^{\circ}, \theta, \psi\right)$, the transformation matrix $\mathbf{T}$ is

$$
[\mathbf{T}]=\left[\begin{array}{lccc}
-c & -d & 0 & X \\
b d & -b c & a & Y \\
-a d & a c & b & Z \\
0 & 0 & 0 & 1
\end{array}\right]
$$

where

$$
a=\sin \theta ; b=\cos \theta ; c=\sin \psi ; d=\cos \psi
$$

Using the same method above, the equations of the three normal planes can be obtained. 


$$
\begin{gathered}
\left(a c R_{b}-Z\right) y+\left(b c R_{b}+Y\right) z=0 \\
\sqrt{3}\left(\sqrt{3} a d R_{b}-a c R_{b}-2 Z\right) x+\left(\sqrt{3} a d R_{b}-2 Z-a c R_{b}\right) y+\left(2 Y+2 \sqrt{3} X+\sqrt{3} b d R_{b}-3 c R_{b}-\right. \\
\left.\sqrt{3} d R_{b}-b c R_{b}-6 R_{a} \cos \left(\beta_{0} / 2\right)\right) z+3 R_{a} \cos \left(\beta_{0} / 2\right)\left(2 Z+a c R_{b}-\sqrt{3} a d R_{b}\right)=0 \\
\sqrt{3}\left(a c R_{b}+2 Z+\sqrt{3} a d R_{b}\right) x-\left(2 Z+\sqrt{3} a d R_{b}+a c R_{b}\right) y+\left(2 Y-b c R_{b}-6 R_{a} \cos \left(\beta_{0} / 2\right)-\right. \\
\left.\sqrt{3} b d R_{b}-3 c R_{b}+\sqrt{3} d R_{b}-2 \sqrt{3} X\right) z+3 R_{a} \cos \left(\beta_{0} / 2\right)\left(2 Z+a c R_{b}+\sqrt{3} a d R_{b}\right)=0
\end{gathered}
$$

The equation of plane $B_{1} B_{3} B_{5}$ is

$$
a y+b z-a Y-b Z=0
$$

Solving Eqs. (27), (28) and (29) for $x, y$ and $z$, and then substituting them into Eq. (30), the singularity equation is

$$
[(\sin \theta) Y+(\cos \theta) Z]\left(e Z^{2}-f X Z+g Y Z+h X-i Y+j Z+k\right)=0
$$

Eq. (31) shows that the singular loci include a plane and a conicoid. The plane equation is the same as Eq. (25). It also represents that in this case all the six lines cross a common line. This case belongs to the first special-linear-complex singularity. The quadratic equation is

$$
e Z^{2}-f X Z+g Y Z+h X-i Y+j Z+k=0
$$

Eq. (32) is a singularity equation with respect to the fixed frame $O-X Y Z$. When the mobile shown in Fig. 5 rotates an angle $\psi$ about $Z$ "-axis again, its orientation is $\left(90^{\circ}, \theta, \psi\right)$.

The plane in which the mobile lies is still $\theta$-plane. After the coordinate transformation, the equation of the singularity curve in $\theta$-plane with respect to the frame $\mathrm{O}_{2}-x y z$ is

$$
\left\{\begin{array}{l}
2(\sin \psi) y^{2}+2(\cos \psi) x y+R_{b} \sin (2 \psi) x+\left(-2 u \sin \psi+6 R_{a} \sin \psi \cos \left(\beta_{0} / 2\right)\right. \\
\left.-R_{b} \cos (2 \psi)\right) y-R_{b}^{2} \sin \psi+R_{b} \cos (2 \psi)\left(3 R_{a} \cos \left(\beta_{0} / 2\right)-u\right)=0 \\
z=0
\end{array}\right.
$$

It is also a hyperbola. In addition, Eq. (33) is independent of the Euler angle $\theta$.

\subsubsection{Analysis of the Singularity Property}

The four invariants $\Delta, D, I$ and $J$ of Eq. (32) are

$$
\Delta=\left|\begin{array}{cccc}
0 & 0 & -\frac{f}{2} & \frac{h}{2} \\
0 & 0 & \frac{g}{2} & -\frac{i}{2} \\
-\frac{f}{2} & \frac{g}{2} & e & \frac{j}{2} \\
\frac{h}{2} & -\frac{i}{2} & \frac{j}{2} & k
\end{array}\right|=\frac{R_{b}^{2} \sin ^{6} \theta \cos ^{2} 3 \psi}{4} \geq 0
$$




$$
\begin{gathered}
D=\left|\begin{array}{llc}
0 & 0 & -\frac{f}{2} \\
0 & 0 & \frac{g}{2} \\
-\frac{f}{2} & \frac{g}{2} & e
\end{array}\right|=0 \\
I=2 \sin \psi(1+\cos \theta), J=-\sin ^{2} \theta
\end{gathered}
$$

The following cases are discussed according to its invariants, in which $D$ is always zero whatever $\theta$ and $\psi$ are.

1. If $\theta \neq 0, \psi \neq \pm 30^{\circ}, \pm 90^{\circ}$, and $\pm 150^{\circ}$, then $D=0, \Delta>0$, the singular locus denoted by Eq. (32) is a hyperbolic paraboloid. Generally, the six lines 1, 2, ., 6 belong to a general linear complex when point $P$ locates at the surface.

2. If $\theta=0$, Eq. (32) can be written as

$$
4(\sin \psi) Z^{3}=0
$$

a. When $\psi=0$ and $Z \neq 0$, namely, the orientation is $\left(90^{\circ}, 0,0\right)$, Eq. (37) is an identical equation and the mechanism is singular whatever the position of point $P$ in threedimensional space is. This is the Fichter's singular configuration and all the six lines belong to a general linear complex.

b. When $Z=0$, the moving platform and the base are coplanar. The mechanism is also singular whatever Euler angle $\psi$ is. The mechanism holds three remnant freedoms when all the legs are locked. In this case, there exist the first and the second speciallinear-complex singularities.

3. If $\theta \neq 0, \psi= \pm 30^{\circ}, \pm 90^{\circ}$, or $\pm 150^{\circ}$, then $D=0, \Delta=0$ and $J \neq 0$, and the conicoid degenerates into a pair of intersecting planes. For instance, when $\psi= \pm 30^{\circ}$, two equations are

$$
\begin{gathered}
2 Z-R_{b} \sin \theta=0 \\
\sqrt{3}(\sin \theta) X-(\sin \theta) Y-(1+\cos \theta) Z-R_{b} \sin \theta+3 R_{a} \sin \theta \cos \left(\beta_{0} / 2\right)=0
\end{gathered}
$$

When $\psi=-30^{\circ}, \pm 90^{\circ}$, or \pm 150 , the conicoid also degenerates into two planes. The singularity cases are similar to the above.

\subsubsection{Analysis of Other Singularities}

The singularities discussed above are all for the orientations, $\left( \pm 90^{\circ}, \theta, \psi\right)$, of the mobile. In these cases, the intersecting lines between the oblique moving plane and the basic one are parallel to line $C_{1} C_{2}$ or $A_{1} A_{5}$, one of the three sides of the triangle $A_{1} A_{3} A_{5}$.

The similar singularities with a plane equation and a quadratic one can also occur when the orientations are as follows

1. The Euler angles are

$$
\left(-150^{\circ}, \theta, \psi\right) \text { or }\left(30^{\circ}, \theta, \psi\right)
$$

All the intersecting lines between the oblique mobile and the base are parallel to line $C_{3} C_{4}$ or $A_{1} A_{3}$.

2. The Euler angles are

$$
\left(150^{\circ}, \theta, \psi\right) \text { or }\left(-30^{\circ}, \theta, \psi\right)
$$


All the intersecting lines between the oblique mobile and the base are parallel to line $C_{5} C_{6}$ or $A_{3} A_{5}$.

For the two cases, the singularity equation can also resolve into two parts: one is a plane equation containing the corresponding side $C_{i} C_{j}$, another is a hyperbolic paraboloid equation, too. When $\psi= \pm 30^{\circ}, \pm 90^{\circ}$, or $\pm 150^{\circ}$, the hyperbolic paraboloid also degenerates into two planes.

However, when the orientation is

$$
\left(\phi, \theta, \pm 30^{\circ}\right),\left(\phi, \theta, \pm 90^{\circ}\right) \text { or }\left(\phi, \theta, \pm 150^{\circ}\right)
$$

in which $\phi$ and $\theta$, can be arbitrary values, the singularity locus also consists of two parts: One is a plane; another is also a hyperbolic paraboloid. When point $P$ translates in the plane, two of three points $B_{1}, B_{3}$ and $B_{5}$ lie in the basic plane.

\subsection{Singularity distribution in three-dimensional space}

According to the analysis method above, we may easily know the distribution characteristics of the singularity loci of the 3/6-Gough-Stewart manipulator, and draw their singularity surface in three-dimensional space for some different orientations of the mobile in frame $O-X Y Z$. Here, the parameters of the mechanism are set to $R_{a}=\sqrt{2}, R_{m}=1 \mathrm{~m}$ and $\beta_{0}=90^{\circ}$, and the surfaces are shown in Fig. 6 .

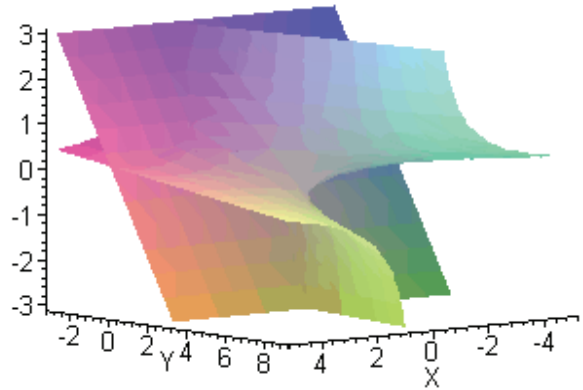

(a) The orientation $\left(90^{\circ} 45^{\circ} 0\right)$

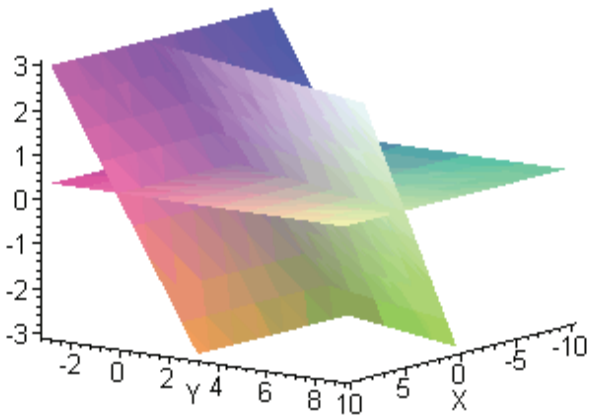

(c) The orientation $\left(90^{\circ} 45^{\circ} 30^{\circ}\right)$

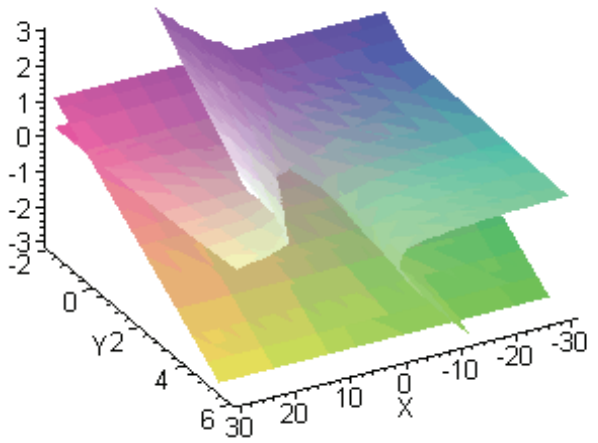

(b) The orientation $\left(90^{\circ} 30^{\circ} 60^{\circ}\right)$

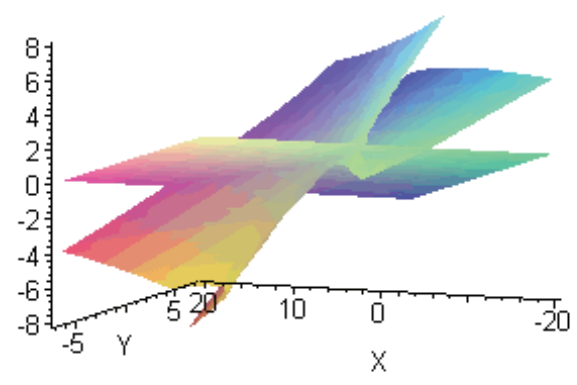

(d) The orientation $\left(45^{\circ} 25^{\circ} 30^{\circ}\right)$

Fig. 6. The singularity loci for 3/6-Stewart parallel manipulator 
The readers may wonder that the singularity loci are so huge and completed and ask how can the GSP work? In practice, if you notice the position of the origin point of the O-XYZ system in Figures, and the magnitudes of the parameters, $R_{a}=\sqrt{2}$ and $R_{m}=1 \mathrm{~m}$, you can find that the workspace of the manipulator is smaller relative the singularity loci shown in figures. You can easily design the manipulator making its workspace locate over the singularity loci and avoiding singularity.

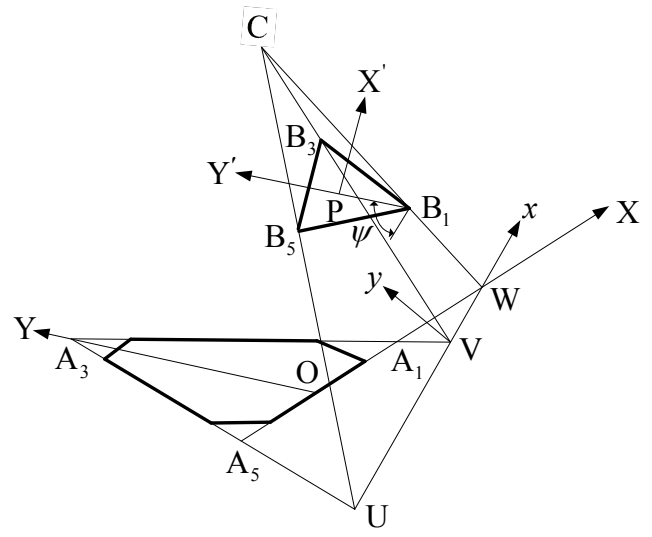

Fig. 7. The general case

\section{Structure and property of the singularity loci of the 3/6-Gough-Stewart for general orientations}

When $\phi$ takes any value with the exception of $\pm 30^{\circ}, \pm 90^{\circ}$, or $\pm 150^{\circ}$, this is the general orientation case of the mobile of the GSP, and the analysis of the singularity loci is more difficult. In this case, UV is not parallel to any side of triangle $A_{1} A_{3} A_{5}$, as shown in Fig. 7.

\subsection{Singularity equation based on Theorem 3 for general orientations}

For the most general orientations of the mobile, $\phi \neq \pm 30^{\circ}, \pm 90^{\circ}$, and $\pm 150^{\circ}$, the singularity equation can be directly obtained by using Theorem 3 . The equation of normal plane $B_{1} C_{1} C_{2}$ is

$$
\left|\begin{array}{ccc}
x^{\prime}-B_{1 x} & y^{\prime}-B_{1 y} & z^{\prime}-B_{1 z} \\
C_{1 x}-B_{1 x} & C_{1 y}-B_{1 y} & C_{1 z}-B_{1 z} \\
C_{2 x}-B_{1 x} & C_{2 y}-B_{1 y} & C_{2 z}-B_{1 z}
\end{array}\right|=0
$$

where $\left(x^{\prime}, y^{\prime}, z\right)$ denotes coordinates of the moving point on plane $B_{1} C_{1} C_{2}$ in the fixed frame. This gives

$$
F y^{\prime}+G z^{\prime}=0
$$

Similarly, equations of three planes $B_{3} C_{3} C_{4}, B_{5} C_{5} C_{6}$ and $B_{1} B_{3} B_{5}$ can be obtained as well. According to Theorem 3, solving the linear equation system of the four planes for intersecting point $C$, the singularity locus equation for general orientations is as follows 


$$
\begin{aligned}
& \mathrm{f}_{1} Z^{3}+\mathrm{f}_{2} X Z^{2}+\mathrm{f}_{3} \mathrm{YZ}^{2}+\mathrm{f}_{4} X^{2} Z+\mathrm{f}_{5} \mathrm{Y}^{2} Z+\mathrm{f}_{6} X Y Z+\mathrm{f}_{7} Z^{2}+\mathrm{f}_{8} X^{2} \\
& +\mathrm{f}_{9} \mathrm{Y}^{2}+\mathrm{f}_{10} X Y+\mathrm{f}_{11} X Z+\mathrm{f}_{12} Y Z+\mathrm{f}_{13} Z+\mathrm{f}_{14} X+\mathrm{f}_{15} \mathrm{Y}+\mathrm{f}_{16}=0
\end{aligned}
$$

where $(X, Y, Z)$ are the coordinates of center point $P$. It is a polynomial expression of degree three. The equation is still very complicated and difficult to further analyze, but it is very simple in the following special cases.

When $\phi \neq \pm 30^{\circ}, \pm 90^{\circ}$, and $\pm 150^{\circ}$ and $\psi$ is one of the values $\pm 30^{\circ}, \pm 90^{\circ}$, or $\pm 150^{\circ}$, Eq. (42) degenerates into a plane and a hyperbolic paraboloid as well. For example, when $\psi=90^{\circ}$, the singularity equation is

$$
\begin{aligned}
& \left(2 Z+R_{b} \sin \theta\right)\left(a_{11} X^{2}+a_{22} Y^{2}+a_{33} Z^{2}+2 a_{23} Y Z+2 a_{31} Z X+2 a_{12} X Y+\right. \\
& \left.2 a_{14} X+2 a_{24} Y+2 a_{34} Z+a_{44}\right)=0
\end{aligned}
$$

where these coefficients are listed in the Appendix 2. Eq. (43) indicates a plane and a hyperbolic paraboloid. The first factor forms a plane equation

$$
2 \mathrm{Z}+\mathrm{R}_{\mathrm{b}} \sin \theta=0
$$

which is parallel to the basic plane. When point $P$ lies in the plane, the mechanism is singular for orientation $\left(\phi, \theta, 90^{\circ}\right)$, because points $B_{3}$ and $B_{5}$ lie in the basic plane. This is similar to Case 6. All the six lines cross the same line $C_{1} C_{2}$.

\subsection{Singularity analysis using singularity-equivalent-mechanism}

The singularity locus expression (Eq. (43)) for general orientations has been derived by Theorem 3. But it is still quite complicated, and we are not sure whether it consists of some typical geometrical figures. Meanwhile the property of singularity loci is unknown yet. In order to reply this question, a "Singularity-Equivalent-Mechanism" which is a planar mechanism is proposed. Thus the troublesome singularity analysis of the GSP can be transformed into a position analysis of the simpler planar mechanism.

\subsubsection{The parallel case}

\subsubsection{The Singularity-Equivalent-Mechanism}

In the parallel case, the three Euler angles of the mobile platform are $\left(90^{\circ}, \theta, \psi\right)$, while $\theta$ and $\psi$ can be any nonzero value. The mobile plane of the mechanism lies on $\theta$-plane (Fig. 5).

The corresponding imaginary planar singularity-equivalent-mechanism is illustrated in Fig. 8. Where Rdenotes a revolute pair and $\mathrm{P}$ a prismatic pair, triangle $\mathrm{B}_{1} \mathrm{~B}_{3} \mathrm{~B}_{5}$ is connected to ground by three kinematic chains, RPP, PPR and RPR. The latter two pass through two points $U$ and $V$, respectively, while the first one slides along the vertical direction and keeps $\mathrm{L}_{1} \mathrm{C} / / \mathrm{UV}$. Three slotted links, $\mathrm{L}_{1}, \mathrm{~L}_{2}$ and $\mathrm{L}_{3}$, intersect at a common point $\mathrm{C}$. In order to keep the three links always intersecting at a common point and satisfying Deduction 2, a concurrent kinematic chain PRPRP is used. It consists of five kinematic pairs, where two $\mathrm{R}$ pairs connect three sliders. The three sliders and three slotted links form three $P$ pairs. The PRPRP chain coincides with a single point $C$ from top view. Based on the Grübler-Kutzbach criterion, the mobility of the mechanism is two.

It is evident that the planar mechanism can guarantee that the three lines passing through three vertices intersect at a common point, and these three lines can always intersect the 
corresponding sides of the basic triangle. From Deduction 2, every position of the planar mechanism corresponds to a special configuration of the original GSP. So we call it a "singularity-equivalent-mechanism". Thus the position solution of the planar mechanism expresses the singularity of the original mechanism.

\subsubsection{Forward Position Analysis of the Singularity -Equivalent-Mechanism}

The frames are set as the same as in Fig. 5 and Fig. 10. The coordinates of point $\mathrm{P}$ in frame $\mathrm{O}_{2}-x y$ are $(x, y) . \psi$ indicates the orientation of the triangle $\mathrm{B}_{1} \mathrm{~B}_{3} \mathrm{~B}_{5}$ in $\theta$,-plane. In order to obtain the locus equation of point $P$, firstly we can set three equations of three lines passing through the three vertices, and substitute the coordinates of points $B_{1}, B_{3}$ and $B_{5}$ into the equations, then $(x, y)$ and $\psi$ can be obtained.

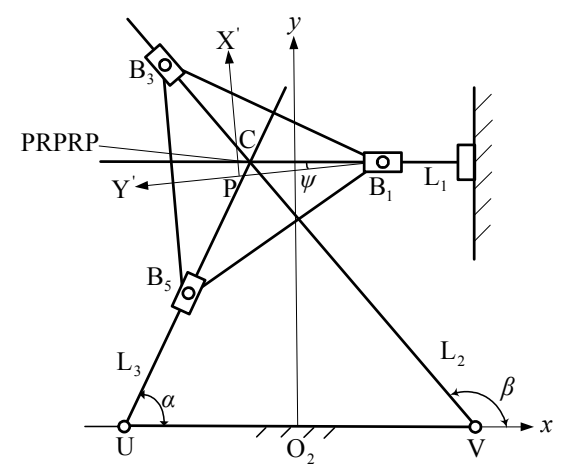

Fig. 8. The singularity-equivalent-mechanism for $\left(90^{\circ}, \theta, \psi\right)$

Considering that the mobility of this mechanism is two, there need two inputs $\alpha$ and $\beta$. Three equations of three lines $\mathrm{CU}, \mathrm{CV}$ and $\mathrm{CB}_{1}$ in reference frame $\mathrm{O}_{2}-x y$ are respectively

$$
\begin{aligned}
& \mathrm{Y}=(\tan \alpha)(\mathrm{X}+\mathrm{a} / 2) \\
& \mathrm{Y}=(\tan \beta)(\mathrm{X}-\mathrm{a} / 2)
\end{aligned}
$$

and

$$
\mathrm{Y}=-\frac{\mathrm{a} \tan \mathrm{atan} \beta}{\tan \alpha-\tan \beta}
$$

Solving Eqs. (52), (53) and (54) yields

$$
\begin{gathered}
x=\frac{R_{b} \cos \psi J_{1}-\left(\sqrt{3} R_{b} \sin \psi+a\right) J_{3}}{2(\tan a-\tan \beta)} \\
y=\frac{R_{b} \sin \psi J_{2}-\sqrt{3} R_{b} J_{3} \cos \psi-2 a \tan a \tan \beta}{2(\tan a-\tan \beta)}
\end{gathered}
$$

and

$$
\tan \psi=\frac{(\tan \beta+\tan \alpha)}{\sqrt{3} \tan \alpha-\sqrt{3} \tan \beta-2 \tan \alpha \tan \beta}
$$


where $J_{1}=\tan a-\tan \beta-2 \sqrt{3}, J_{2}=\tan a-\tan \beta-2 \sqrt{3} \tan a \tan \beta, J_{3}=\tan \alpha+\tan \beta$, Eqs. (48), (49) and (50) denote direct kinematics of the mechanism.

\section{2. 1. 3. Singularity Equation in the $\theta$ - plane}

Once the orientation $\left(90^{\circ}, \theta, \psi\right)$ of the mobile platform is specified, in Fig.10, Euler angle $\psi$ is an invariant. So it only needs to choose one input in this case. From Eq. (65) one obtains

$$
\tan \beta=\frac{\tan \alpha(\sqrt{3} \tan \psi-1)}{\sqrt{3} \tan \psi+2 \tan \alpha \tan \psi+1}
$$

So the singularity equation in $\theta$ - plane for the orientation $\left(90^{\circ}, \theta, \psi\right)$ can be obtained from Eqs. (48), (49) and (50) by eliminating parameters $\alpha$ and $\beta$

$$
\begin{aligned}
& 2(\sin \psi) y^{2}+2(\cos \psi) x y+R_{b} \sin (2 \psi) x+ \\
& \left(\sqrt{3} a \sin \psi-R_{b} \cos (2 \psi)\right) y-R_{b}^{2} \sin \psi+\sqrt{3} a R_{b} \cos (2 \psi) / 2=0
\end{aligned}
$$

where $a=2\left(3 R_{a} \cos \left(\beta_{0} / 2\right)-u\right) / \sqrt{3}$. Eq. (52) denotes a hyperbola. Especially, when $\psi= \pm 90^{\circ}$, Eq. (52) degenerates into a pair of intersecting straight lines respectively. Two of the four equations are

$$
\mathrm{y}-\mathrm{R}_{\mathrm{b}} / 2=0, \mathrm{y}+\mathrm{R}_{\mathrm{b}} / 2=0
$$

In both cases, two points $B_{3}$ and $B_{5}$ lie in line UV. So that four lines are coplanar with the base plane. This is the singularity of Case 6 . The similar situation is for $\psi=30^{\circ}, \psi=-150^{\circ}$, $\psi=-30^{\circ}$ and $\psi=150^{\circ}$.

\subsubsection{The general case}

When $\phi \neq \pm 30^{\circ}, \pm 90^{\circ}$, and $\pm 150^{\circ}$, the intersecting line UVW between $\theta$ - plane and the base one is not parallel to any side of triangle $\mathrm{A}_{1} \mathrm{~A}_{3} \mathrm{~A}_{5}$. This is the most general and also the most difficult case.

\subsubsection{The Singularity-Equivalent-Mechanism}

Fig. 11 shows the singularity-equivalent-mechanism. The triangle $B_{1} B_{3} B_{5}$ is connected to the ground passing through three points $\mathrm{W}, \mathrm{V}$ and $\mathrm{U}$ by three RPR kinematic chains. The three points $\mathrm{U}, \mathrm{V}$ and $\mathrm{W}$, as shown in Fig. 9, are three intersecting points between $\theta$-plane and sides $A_{3} A_{5}, A_{1} A_{3}$ and $A_{1} A_{5}$, respectively. Three slotted links $L_{1}, L_{2}$ and $L_{3}$ intersect at a common point $\mathrm{C}$. In order to keep the three links always intersecting at a common point, a concurrent kinematic chain, PRPRP, is used as well. Therefore, all the configurations of the equivalent mechanism satisfying Deduction 2 are special configurations of the GoughStewart manipulator. So we can analyze direct kinematics of the equivalent mechanism to find singularity loci of the manipulator.

Similarly the mobility of the equivalent mechanism is two, and it needs two inputs when analyzing its position.

\subsubsection{Forward Position Analysis of the Singularity-Equivalent -Mechanism}

The frames are set as shown in Fig. 11. Similar to section 4.2.1.2, we may set three equations of three straight lines passing through three vertices, and substitute the coordinates of points $B_{1}, B_{3}$ and $B_{5}$ into the equations, then solutions, $(x, y)$ and $\psi$, can be obtained 


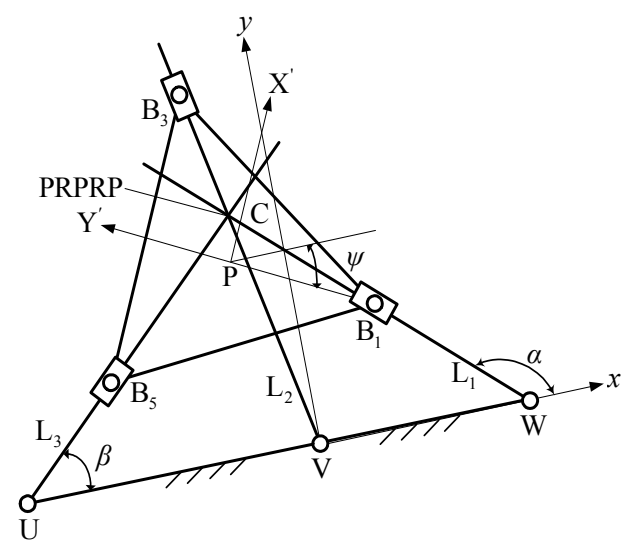

Fig. 9. The singularity-equivalent-mechanism for general case

$$
\begin{gathered}
x=-\left(3 R_{b} \sin \psi-2 R_{b} \tan \alpha \cos \psi+2 w \tan \alpha+\sqrt{3} R_{b} \cos \psi\right. \\
\left.-R_{b} \tan \beta \cos \psi+\sqrt{3} R_{b} \tan \beta \sin \psi-2 u \tan \beta\right) /(2 \tan \beta-2 \tan \alpha) \\
\mathrm{y}=\left(-\mathrm{R}_{\mathrm{b}} \tan \alpha \sin \psi-\sqrt{3} \mathrm{R}_{\mathrm{b}} \tan \alpha \cos \psi+3 \mathrm{R}_{\mathrm{b}} \tan \alpha \tan \beta \cos \psi+2 \mathrm{u} \tan \alpha \tan \beta\right. \\
\left.-\sqrt{3} \mathrm{R}_{\mathrm{b}} \tan \alpha \tan \beta \sin \psi-2 \mathrm{R}_{\mathrm{b}} \tan \beta \sin \psi-2 \mathrm{w} \tan \alpha \tan \beta\right) /(2 \tan \beta-2 \tan \alpha) \\
\tan \psi=\frac{2 \sqrt{3} w \tan \alpha-3 u \tan \alpha \tan \beta-\sqrt{3} u \tan \beta}{\tan \beta(-2 \sqrt{3} w \tan \alpha+\sqrt{3} u \tan \alpha-3 u)}
\end{gathered}
$$

where $u$ indicates the distance from point $\mathrm{U}$ to $\mathrm{V}$, and $w$ the distance from $\mathrm{V}$ to $\mathrm{W}$. Substituting Eq. (56) into Eqs. (55) and (54), and eliminating $\psi$, the relations between $(x, y)$ and the inputs $\alpha, \beta$ can be obtained. This is direct kinematics of the equivalent mechanism.

\subsubsection{Singularity Equation in the $\theta$ - plane}

Under a general case, Euler angle $\phi$ can be any value with the exception of $\pm 30^{\circ}, \pm 90^{\circ}$, or $\pm 150^{\circ}$. From Eq (56) one obtains

$$
\tan \beta=\frac{2 \sqrt{3} \mathrm{w} \tan \alpha}{-2 \sqrt{3} \mathrm{w} \tan \alpha \tan \psi+\sqrt{3} \mathrm{u} \tan \alpha \tan \psi-3 \mathrm{u} \tan \psi+3 \mathrm{u} \tan \alpha+\sqrt{3} \mathrm{u}}
$$

In the case of some specified $\psi$, there are the same three particular situations that is $\mathrm{B}_{1}$ and $B_{5}, B_{1}$ and $B_{3}$, or $B_{3}$ and $B_{5}$ lie in the line $U V$, respectively. The singularity loci are three pairs of intersecting straight lines.

In order to use the above-mentioned formulas, $u$ and $w$ in Eq. (57) should be calculated in advance. They depend on their relative positions in UV, as shown in Fig. 10.

The distance $w$ between $\mathrm{V}$ and $\mathrm{W}$ is

$$
|w|=|\mathrm{WV}|=\left|\frac{3 R_{a} \cos \left(\beta_{0} / 2\right)-\sqrt{3} x_{V}}{\cos \phi}\right|
$$




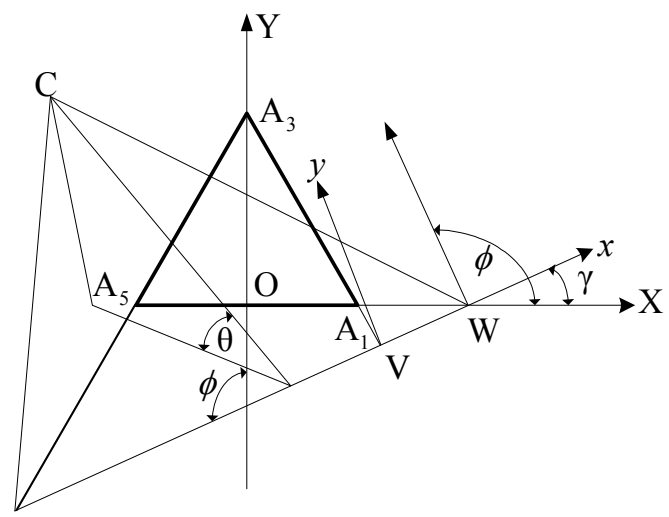

$\mathrm{U}$

Fig. 10. The Intersecting Line UW of two planes

The distance $u$ between $U$ and $\mathrm{V}$ is

$$
|\mathrm{u}|=|U V|=\left|\frac{2 \sqrt{3} \mathrm{x}_{\mathrm{V}}}{(\sqrt{3}+\cot \phi) \sin \phi}\right|
$$

The sign of $w$ is positive when point $\mathrm{W}$ is on the right side of $\mathrm{V}$, and it is negative when $\mathrm{W}$ is on the left side of $\mathrm{V}$. It is similar for the sign of $u$.

For a given $x_{v}$, the singularity equation in $\theta$-plane can be obtained by eliminating the parameter $\alpha$

$$
b x y+c y^{2}+d x+e y+f=0
$$

The two invariants $D, \delta$ of Eq. (60) are

$$
D=\left|\begin{array}{ccc}
0 & b / 2 & d / 2 \\
b / 2 & c & e / 2 \\
d / 2 & e / 2 & f
\end{array}\right|=-\frac{1}{4}\left(b^{2} f+d^{2} c-b d e\right)
$$

and

$$
\delta=\left|\begin{array}{cc}
0 & b / 2 \\
b / 2 & c
\end{array}\right|=-\frac{1}{4} b^{2}<0
$$

Generally, $D \neq 0$ and $\delta<0$ for a general value of $x_{v}$, so Eq. (60) indicates a set of hyperbolas.

\subsection{Five special cases of the singularity equation}

There are five special cases. For the given parameters $\left(R_{a}, R_{b}, \beta_{0}\right)$ and $(\phi, \theta, \psi), D$ is a quartic equation while $\delta$ a quadratic equation with respect to the single variable $x_{v}$, respectively. Generally, there are four real roots of $x_{v}$ when $D=0$ and $\delta \neq 0$, and Eq. (60) degenerates into four pairs of intersecting straight lines. For the same reason, there is one real root of multiplicity 2 when $\delta=0$ and $D \neq 0$, and Eq. (60) degenerates into a parabola. 


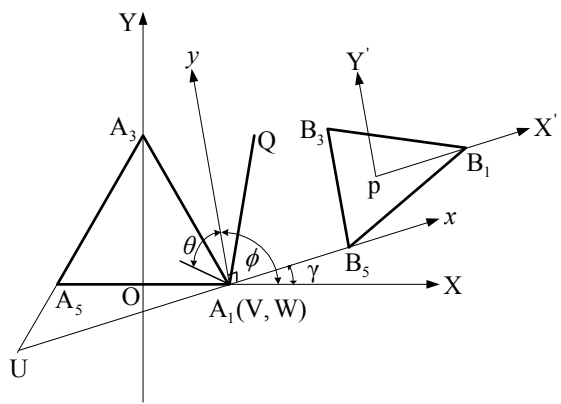

(a) $\mathrm{B}_{5}$ does not coincide with $\mathrm{A} 1$

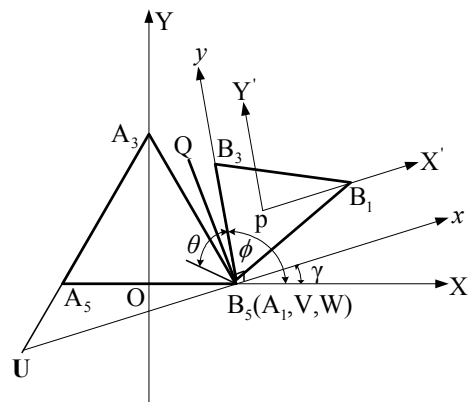

(b) $B_{5}$ coincides with $A_{1}$

Fig. 11. UV passes through the points $A_{1}$

Case 1. The line UV passes through point $A_{1}$, as shown in Fig. 11. In this case $x_{v}=\sqrt{3} R_{a} \cos \left(\beta_{0} / 2\right)$, two points $W$ and $V$ coincide with point $A_{1}$. The singularity equation denoted by Eq. (75) degenerates into a pair of intersecting straight lines

$$
\left[y-R_{b} \sin \left(\psi+60^{\circ}\right)\right]\left[(-\sqrt{3} \sin (\psi)+\cos (\psi)) x+(\sqrt{3} \cos (\psi)+\sin (\psi)) y+R_{b}\right]=0
$$

One of them is

$$
y-R_{b} \sin \left(\psi+60^{\circ}\right)=0
$$

Case 2. UV passes through point $\mathrm{A}_{3}$. In this case $x_{v}=0$, two points $\mathrm{U}$ and $\mathrm{V}$ coincide with point $A_{3}$. Eq. (60) degenerates into a pair of intersecting straight lines either

$$
\left[\left(y+R_{b} \sin (\psi)\right]\left[x \cos (\psi)+y \sin (\psi)-R_{b} / 2\right]=0\right.
$$

The first part of Eq. (65) indicates a straight line parallel to $x$-axis. Similarly when $\mathrm{B}_{1}$ coincides with point $\mathrm{A}_{3}$, the singularity of this point is the first special-linear-complex singularity and the instantaneous motion is a pure rotation. When $B_{1}$ does not coincide with $\mathrm{A}_{3}$, the singularities of points lying in this straight line are the general-linear-complex singularity and its instantaneous motion is a twist with $h^{m \neq} \neq 0$.

The second part of Eq. (62) denotes another straight line. The singularities of points lying in this straight line are all the general-linear-complex singularity.

Case 3. UV passes point $A_{5}$. In this case

$$
x_{v}=\sqrt{3} R_{a} \cos \left(\beta_{0} / 2\right)(\sqrt{3}+\cot \phi) /(\sqrt{3}-\cot \phi)
$$

two points $U$ and $W$ coincide with point $A_{5}$. Eq. (60) degenerates into a pair of intersecting straight lines.

$$
\begin{aligned}
& {\left[y-R_{b} \sin \left(\psi-60^{\circ}\right)\right][(\sqrt{3} \sin (\psi)+\cos (\psi)) x+(-\sqrt{3} \cos (\psi)+\sin (\psi)) y} \\
& \left.+R_{b}-2 \sqrt{3} \cos \left(\beta_{0} / 2\right) \sin \left(\psi+60^{\circ}\right) / \sin \left(\varphi-60^{\circ}\right)\right]=0
\end{aligned}
$$

The first factor indicates a straight line parallel to the $x$-axis. Similarly when $\mathrm{B}_{3}$ coincides with $A_{5}$, the singularity of this point is the first special-linear-complex singularity. When $B_{3}$ does not coincide with $A_{5}$, the singularities of points lying in this straight line are the general-linear-complex singularity. 
Similarly the second factor of Eq. (66) denotes another straight line. The singularities of points lying in this straight line are all the general-linear-complex singularity.

Case 4. When

$$
x_{v}=\frac{(-1+2 \cos (2 \phi))\left(R_{b} \cos \phi-2 R_{a} \cos \left(\beta_{0} / 2\right) \cos \psi\right)}{(2(\sqrt{3} \sin \phi-\cos \phi) \sin (\phi+\psi))}
$$

Eq. (67) degenerates into a pair of intersecting straight lines as well

$$
\begin{aligned}
& \left(R_{a} \cos \left(\left(\beta_{0}-6 \psi\right) / 2\right)-R_{b} \cos (\phi-2 \psi)+R_{a} \cos \left(\left(\beta_{0}+6 \psi\right) / 2\right)\right. \\
& -2 y \sin (\phi+\psi))(a x+b y+c)=0
\end{aligned}
$$

For the first straight line when $\beta_{0}=90^{\circ},(\phi, \theta, \psi)=\left(60^{\circ}, 30^{\circ}, 0\right)$, and the coordinates of point $\mathrm{P}_{6}$ are $x=R_{b} / 2, y=\left(2 \sqrt{2} R_{a}-R_{b}\right) / 2 \sqrt{3}$, point $B_{5}$ lies in the intersecting line of two normal planes $B_{1} A_{1} A_{5}$ and $B_{3} A_{1} A_{3}$. Therefore, the six lines associated with the six extensible links of the 3/6-GSP intersect a common line $\mathrm{B}_{5} \mathrm{~A}_{1}$. It is the first special-linear-complex singularity. The instantaneous motion is a pure rotation about line $\mathrm{B}_{5} \mathrm{~A}_{1}$. The singularities of points lying in the first line with the exception of the above-mentioned point and the points lying in the second line are all belong to the general-linear-complex singularity.

Case 5. When

$$
x_{v}=R_{a} \cos \left(\beta_{0} / 2\right) \cos \psi(\cos \varphi+\sqrt{3} \sin \phi) / \sin (\phi+\psi), \delta=0 \text { and } D \neq 0,
$$

Eq. (60) degenerates into a parabola.

$$
c y^{2}+d x+e y+f=0
$$

According to the analysis mentioned above, it is shown that the singularity expression in $\theta$ plane is not cubic but always quadratic. This indicates the $\theta$-plane is a very special cross section of the singularity surface, so the special $\theta$-plane can be called the principal section.

Generally speaking, the singularity loci of the 3/6-GSP for the most general orientations are different from those for some special orientations. The singularity loci in infinite parallel principal sections are all quadratic equations. The structure of the singularity loci in the principal sections of the cubic singularity surface includes a parabola, four pairs of intersecting straight lines and infinity of hyperbolas. The singularity loci in threedimensional space are illustrated in Fig. 12.

In addition, it should be pointed out that once the mechanism is singular at the orientation $(\phi, \theta, \psi)$, any orientation with different variable $\theta$ is singular as well (Huang at el. 2003).

\section{Structure and property of the singularity loci of the 6/6-Gough-Stewart}

Base on the above-mentioned analysis of the 3/6-GSP, here we focus on the most difficult issue, the singularity locus analysis of the 6/6-GSP including the singularity equation and the structure of singularity surface. The $6 / 6-$ GSP is typical manipulator. The $6 / 6-G S P$ is represented schematically in Fig. 13. It consists of two semi-regular hexagons: a mobile platform $B_{1} B_{3} \ldots B_{6}$ and a base platform $C_{1} \ldots C_{6}$. They are connected via six extensible prismatic actuators. 


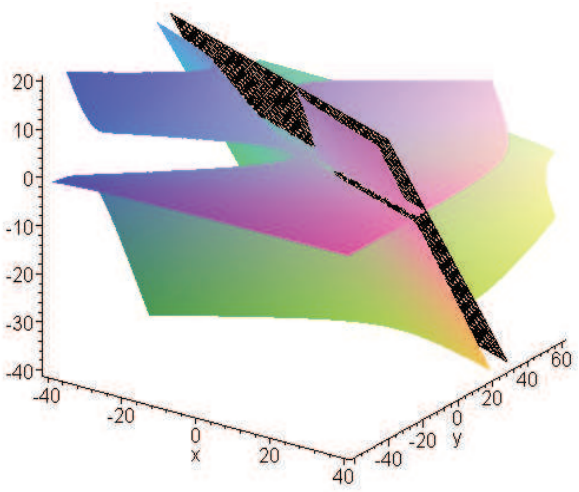

(a) for orientation $\left(60^{0}, 45^{0}, 45^{0}\right)$

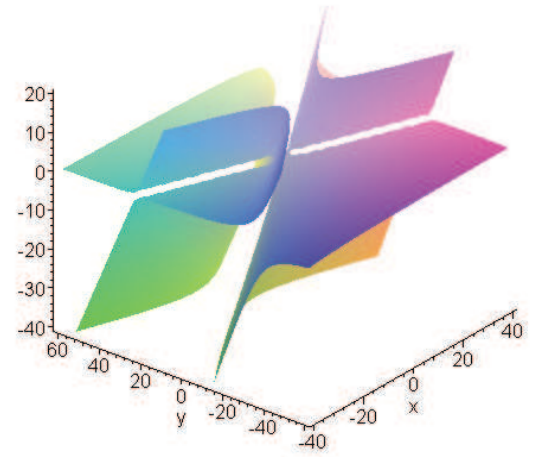

(c) for orientation $\left(60^{0}, 60^{0}, 45^{\circ}\right)$

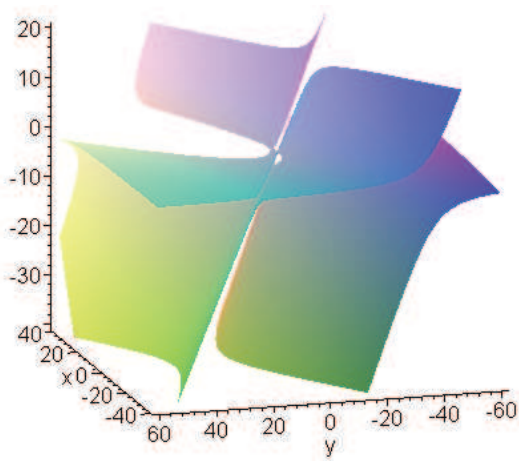

(b) with a principal section $x_{v}=-4$

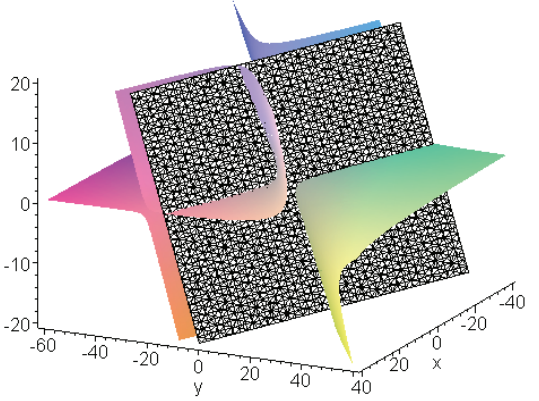

(d) with a principal section $x_{v}=-4$

Fig. 12. The singularity loci in three-dimensional space for the general orientations

\subsection{The Jacobian matrix}

The Jacobian matrix of this class of the Gough-Stewart manipulators can be constructed as follows according to the theory of static equilibrium (Huang and Qu 1987)

$$
\begin{aligned}
{[\mathbf{J}]^{T}=} & {\left[\begin{array}{llllll}
\boldsymbol{S}_{1} & \boldsymbol{\$}_{2} & \boldsymbol{\$}_{3} & \boldsymbol{\$}_{4} & \boldsymbol{S}_{5} & \boldsymbol{\$}_{6}
\end{array}\right]=\left(\begin{array}{cccccc}
\boldsymbol{S}_{1} & \boldsymbol{S}_{2} & \boldsymbol{S}_{3} & \boldsymbol{S}_{4} & \boldsymbol{S}_{5} & \boldsymbol{S}_{6} \\
\boldsymbol{S}_{O 1} & \boldsymbol{S}_{O 2} & \boldsymbol{S}_{O 3} & \boldsymbol{S}_{O 4} & \boldsymbol{S}_{O 5} & \boldsymbol{S}_{O 6}
\end{array}\right) } \\
& =\left(\begin{array}{llllll}
\frac{\left(\boldsymbol{B}_{1}-\boldsymbol{C}_{1}\right)}{\left\|\boldsymbol{B}_{1}-\boldsymbol{C}_{1}\right\|} & \frac{\left(\boldsymbol{B}_{2}-\boldsymbol{C}_{2}\right)}{\left\|\boldsymbol{B}_{2}-\boldsymbol{C}_{2}\right\|} & \frac{\left(\boldsymbol{B}_{3}-\boldsymbol{C}_{3}\right)}{\left\|\boldsymbol{B}_{3}-\boldsymbol{C}_{3}\right\|} & \frac{\left(\boldsymbol{B}_{4}-\boldsymbol{C}_{4}\right)}{\left\|\boldsymbol{B}_{4}-\boldsymbol{C}_{4}\right\|} & \frac{\left(\boldsymbol{B}_{5}-\boldsymbol{C}_{5}\right)}{\left\|\boldsymbol{B}_{5}-\boldsymbol{C}_{5}\right\|} & \frac{\left(\boldsymbol{B}_{6}-\boldsymbol{C}_{6}\right)}{\left\|\boldsymbol{B}_{6}-\boldsymbol{C}_{6}\right\|} \\
\frac{\left(\boldsymbol{C}_{1} \times \boldsymbol{B}_{1}\right)}{\left\|\boldsymbol{B}_{1}-\boldsymbol{C}_{1}\right\|} & \frac{\left(\boldsymbol{C}_{2} \times \boldsymbol{B}_{2}\right)}{\left\|\boldsymbol{B}_{2}-\boldsymbol{C}_{2}\right\|} & \frac{\left(\boldsymbol{C}_{3} \times \boldsymbol{B}_{3}\right)}{\left\|\boldsymbol{B}_{3}-\boldsymbol{C}_{3}\right\|} & \frac{\left(\boldsymbol{C}_{4} \times \boldsymbol{B}_{4}\right)}{\left\|\boldsymbol{B}_{4}-\boldsymbol{C}_{4}\right\|} & \frac{\left(\boldsymbol{C}_{5} \times \boldsymbol{B}_{5}\right)}{\left\|\boldsymbol{B}_{5}-\boldsymbol{C}_{5}\right\|} & \frac{\left(\boldsymbol{C}_{6} \times \boldsymbol{B}_{6}\right)}{\left\|\boldsymbol{B}_{6}-\boldsymbol{C}_{6}\right\|}
\end{array}\right)
\end{aligned}
$$

where vectors, $\boldsymbol{B}_{i}, \boldsymbol{C}_{i}(i=1,2, \ldots, 6)$, respectively denote the vertex vectors of the moving and base platforms with respect to the fixed frame , Fig. 15; $\$_{i}(i=1,2, \ldots, 6)$ is a line vector of the corresponding extensible link, and its Plücker coordinates are as follows $\$_{i}=\left(S_{i} ; S_{O i}\right)=\left(L_{i}, M_{i}\right.$, $\left.N_{i} ; P_{i}, Q_{i}, R_{i}\right)$ where the subscript $i(i=1,2, \ldots, 6)$ indicates the $i$ th limb connected by two vertices $B_{i}, C_{i}$ of the moving and base platforms of the manipulator. $S_{i}$ is a unit vector 
specifying the direction of line vector $\$_{i}$, and $S_{O i}$ is a vector indicating the position of the line vector together with $S_{i}$.

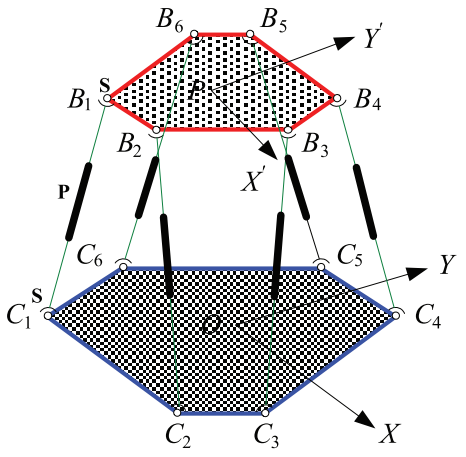

(a) A 6/6-Gough-Stewart manipulators

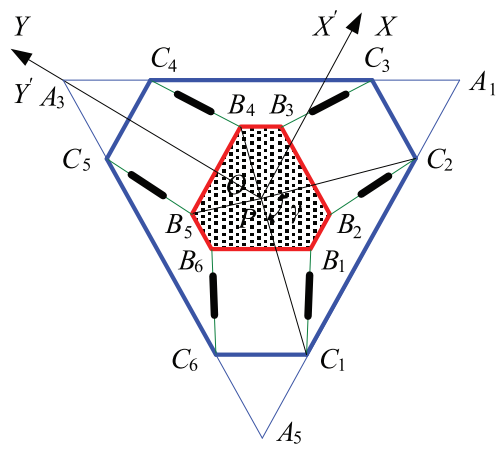

(b) Its top view

Fig. 13. Schematic of a class of the Gough-Stewart manipulators

\subsection{Singularity analysis in three-dimensional space}

A moving reference frame $P-X^{\prime} Y^{\prime} Z^{\prime}$ and a fixed one $O-X Y Z$ are respectively attached to the moving platform and the base platform of the manipulator, as shown in Fig. 15, where origins $P$ and $O$ are corresponding geometric center of the moving and base platforms. The position of the moving platform is given by the position of point $P$ with respect to the fixed frame, designated by $(X, Y, Z)$, and the orientation of the moving platform is represented by the standard Z- $Y-Z$ Euler angles $(\phi, \theta, \psi)$. Furthermore, geometric parameters of the manipulator can be described as follows. The circumcircle radius of the base hexagon is $R_{a}$ and that of the mobile hexagon is $R_{b}$. $\beta_{0}$ denotes the central angle of circumcircles of the hexagons corresponding to sides $\mathrm{C}_{1} \mathrm{C}_{2}$ and $\mathrm{B}_{1} \mathrm{~B}_{6}$, as shown in Fig. 15. The coordinates of six vertices, $B_{i}(i=1,2, \ldots, 6)$, of the moving platform are denoted by $\boldsymbol{B}_{i}{ }^{\prime}$ with respect to the moving frame, and $\boldsymbol{B}_{i}$ with respect to the fixed frame. Similarly, $\boldsymbol{C}_{i}$ and $\boldsymbol{A}_{j}$ represent coordinates of vertices, $C_{i}(i=1,2, \ldots, 6)$ and $\mathrm{A}_{j}(j=1,3,5)$, of the base platform with respect to the fixed frame.

Gosselin and Angeles (1990) pointed out that singularities of parallel manipulators could be classified into three different types, i.e., inverse kinematic singularity, direct kinematic singularity and architecture singularity. Here we only discuss the direct kinematic singularity of this class of 6/6-Gough-Stewart manipulators, which occurs when the determinant of the Jacobian matrix of the manipulator is equal to zero, i.e., $\operatorname{det}(J)=\operatorname{det}\left(J^{T}\right)=0$. Expanding and factorizing the determinant of the Jacobian matrix, the singularity locus equation of the manipulator can be written as

$$
\begin{aligned}
& f_{1} Z^{3}+f_{2} X Z^{2}+f_{3} Y Z^{2}+f_{4} X^{2} Z+f_{5} Y^{2} Z+f_{6} X Y Z+f_{7} Z^{2}+f_{8} X^{2}+ \\
& f_{9} Y^{2}+f_{10} X Y+f_{11} X Z+f_{12} Y Z+f_{13} Z+f_{14} X+f_{15} Y+f_{16}=0
\end{aligned}
$$

Eq. (71) represents the constant-orientation singularity locus of this class of the GoughStewart manipulators in the Cartesian space for a constant orientation $(\phi, \theta, \psi)$. It is a polynomial expression of degree three in the moving platform position parameters $X Y Z$. 
Coefficients of Eq. (71), $f_{i}(i=1,2, \ldots, 15,16)$, are all functions of geometric parameters, $R_{a}, R_{b}$ and $\beta_{0}$, and orientation parameters, $(\phi, \theta, \psi)$, of the manipulator.

Graphical representations of the constant-orientation singularity locus of the manipulator for different orientations are given to illustrate the result, as shown in Fig. 14. Geometric parameters used here are given as $R_{b}=2, R_{a}=1.5, \beta_{0}=\Pi / 2$.

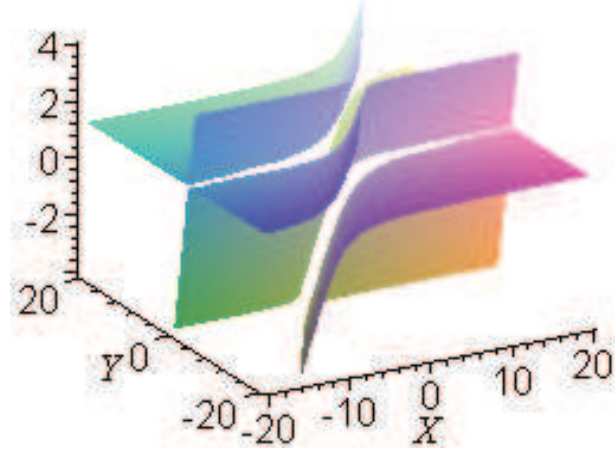

(a) for orientation $\left(90^{\circ}, 60^{\circ}, 30^{\circ}\right)$

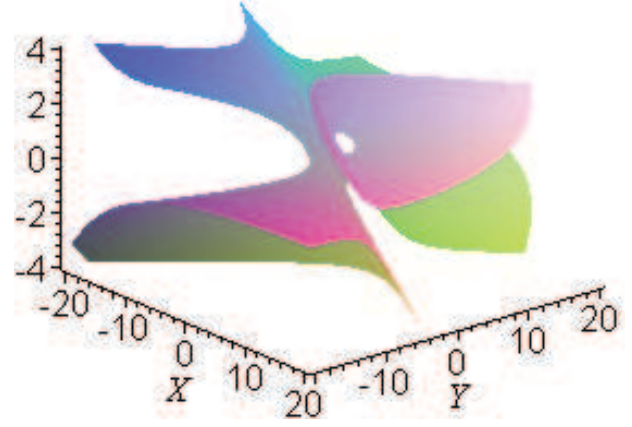

(c) for orientation $\left(60^{\circ}, 30^{\circ}, 45^{\circ}\right)$

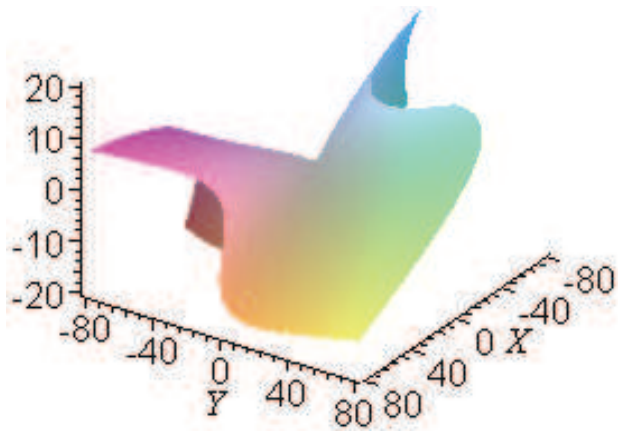

(b) for orientation $\left(-90^{\circ}, 30^{\circ}, 60^{\circ}\right)$

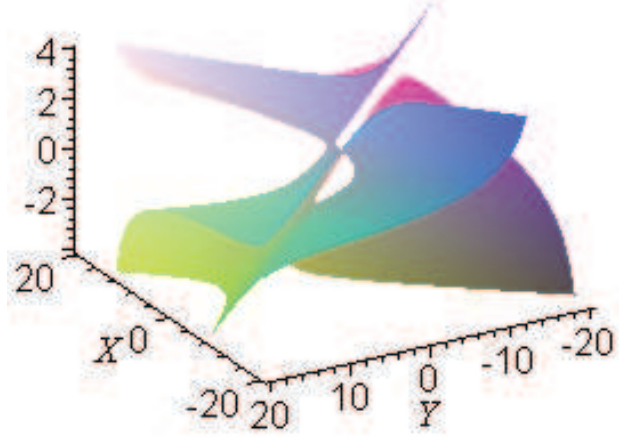

(d) for orientation $\left(45^{\circ}, 30^{\circ}, 45^{\circ}\right)$

Fig. 14. Singularity loci for different orientations

From Figure 14, it can be clearly seen that the singularity loci for different orientations are quite different, and they are complex and various. Among them, the most complicated graph of the singularity loci looks like a trifoliate surface, whose two branches are of the shape of a horn with one hole (Figure 14 (c) and (d)).

\subsection{Singularity analysis in parallel principal-sections 5.3.1 Singularity locus equation in $\theta$-plane}

Huang, Chen and $\mathrm{Li}$ (2003) pointed out that the cross-sections of the cubic singularity locus equation of the 3/6-GSP in parallel $\theta$-planes are all quadratic expressions that include a parabola, four pairs of intersecting lines and infinite hyperbolas. This conclusion is of great importance for the property identification of the singularity loci of the 3/6-GSP. Similarly, in order to identify the characteristics of singularity loci of this class of the 6/6-GSP, singularity loci of the manipulator in parallel $\theta$-planes will also be discussed in this section. Fig. 16 
shows the position of the manipulator for orientation $(\phi, \theta, \psi)$. The oblique plane is $\theta$-plane on which the moving platform lies.

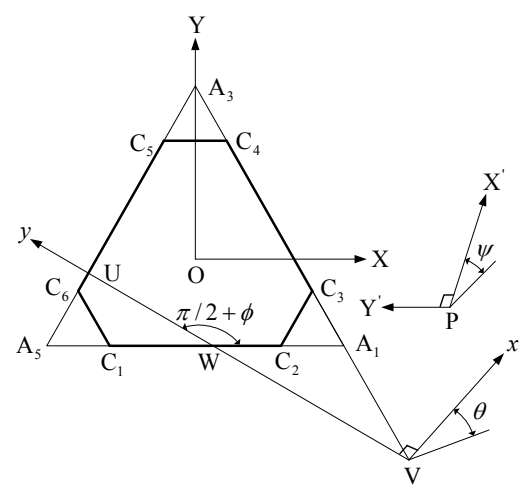

Fig. 15. The position of the manipulator for orientation $(\phi, \theta, \psi)$

When $\theta \neq 0$, the moving platform is not parallel to the base one. $\theta$-plane intersects the base plane at a line $U W V$, where points $U, W, V$ are intersecting points between $\theta$-plane and three sides, $A_{3} A_{5}, A_{1} A_{5}$, and $A_{3} A_{1}$, of the base hexagon, as shown in Fig. 15 . We set another moving reference frame $\mathbf{V}$ - $x y$ in $\theta$-plane, and the coordinates of point $P$ in this moving frame V-xy are denoted by $(x, y)$.

Equations of three lines, $A_{3} A_{5}, A_{1} A_{5}$, and $A_{3} A_{1}$, in the fixed frame $O-X Y Z$ can be easily written. Owing to space limitations, we do not present these equations here. As point $\mathbf{V}$, i.e., origin of the moving frame $\mathbf{V}$ - $x y$, lies on line $\mathrm{A}_{3} \mathrm{~A}_{1}$, and it can be assumed that the coordinates of point $\mathrm{V}$ with respect to the fixed frame $\mathrm{O}-\mathrm{X} Y \mathrm{Z}$ are

$$
\boldsymbol{V}:\left(X_{V}, Y_{V}, 0\right)
$$

where $X_{V}$ is a variable indicating the position of $\theta$-plane, i.e., the position of the moving platform for any given geometric and orientation parameters, and $Y_{V}$ can be established by the following expression

$$
Y_{V}=2 R_{a} \cos \left(\beta_{0} / 2\right)-\sqrt{3} X_{V}
$$

So, the equation of line UV can be written as follows

$$
Y_{U V}-2 R_{a} \cos \left(\beta_{0} / 2\right)+\sqrt{3} X_{V}=-\cot (\phi)\left(X_{U V}-X_{V}\right)
$$

Therefore, coordinates of points $\mathrm{U}$ and $\mathrm{W}$ can be easily obtained. The coordinates of point $P$ designated by $(X, Y, Z)$ with respect to the fixed frame and $(x, y)$ in the moving frame $\mathbf{V}$ - $x y$ satisfy the following expression

$$
\begin{aligned}
& X=\cos \phi \cos \theta x-\sin \phi y+X_{V} \\
& Y=\sin \phi \cos \theta x+\cos \phi y+Y_{V} \\
& Z=-\sin \theta x
\end{aligned}
$$

Substituting Eq. (81) into Eq. (78) and after some rearrangements and factorizations, the singularity locus equation of the manipulator in $\theta$-plane can be written as follows 


$$
\sin ^{3} \theta\left(a x^{2}+2 b x y+c y^{2}+2 d x+2 e y+f\right)=0
$$

Since $\theta \neq 0$, the singularity locus equation of the manipulator with respect to $\theta$-plane becomes

$$
a x^{2}+2 b x y+c y^{2}+2 d x+2 e y+f=0
$$

It can be proved that the coefficient $c$ is always equal to zero, so Eq. (76) is a quadratic polynomial expression with respect to $x$ and $y$, and the maximum degree of variable $x$ is 2 and $y$ is 1 . Coefficients $a, b, d, e, f$ of Eq. (76) are all functions of geometric parameters $R_{a}, R_{b}$ and $\beta_{0}$, Euler angles $(\phi, \psi)$ and $X_{V}$. They are all independent of Euler angle $\theta$. Generally, the intersecting curve between a cubic surface and a plane is also a cubic expression that may also contain a closed-loop curve. For example, when $\left.R_{a}=2, R_{b}=1.5, \beta_{0}=\Pi / 2,(\phi, \theta, \psi\rangle\right)=(\Pi / 3$, $\Pi / 6, \Pi / 4)$, intersecting curves between the corresponding singularity locus surface and the following two planes, $Z=-Y / 3$ and $Z=-4(X-14) / 45$, are respectively presented as follows

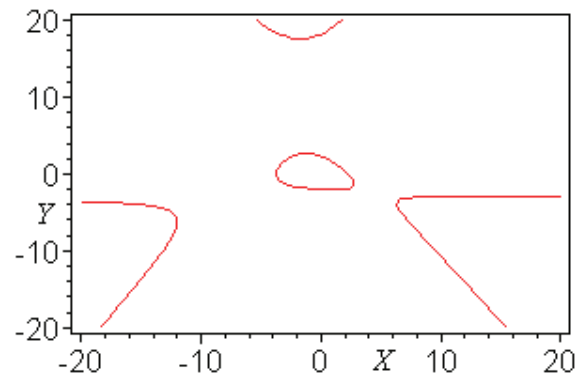

(a) with the plane $Z=-Y / 3$

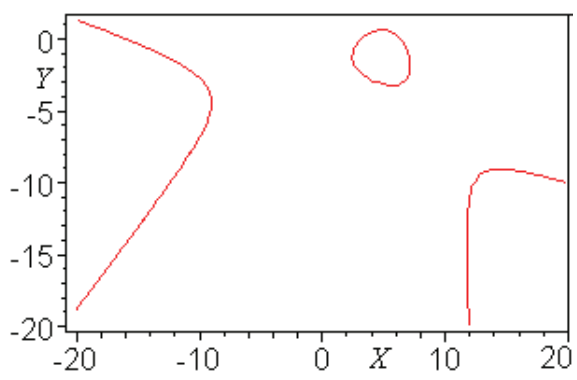

(b) with the plane $Z=-4(X-14) / 45$

Fig. 16. Intersecting curves of closed-loop with different sections

Obviously, the intersecting curves between the singularity locus surface of the manipulator and the two aforementioned planes are actually cubic expressions, which contain a closedloop curve, Fig.18. However, Eq. (76) is always a quadratic polynomial expression; it is worth noting the same conclusion presented above holds for any manipulator of this class of the Gough-Stewart manipulators considered in the current study. Therefore, $\theta$-plane reflects characteristics of the singularity loci of this class of the Gough-Stewart manipulators, and that is why we call it the principal-section.

\subsubsection{Property identification of the singularity loci in parallel principal-sections}

The property of the singularity loci of the manipulator in parallel principal-sections can be analyzed by two invariants, $D$ and $\delta$, of Eq. (76)

$$
\begin{gathered}
\delta=\left|\begin{array}{ll}
a & b \\
b & c
\end{array}\right|=a c-b^{2}=-b^{2} \\
D=\left|\begin{array}{lll}
a & b & d \\
b & c & e \\
d & e & f
\end{array}\right|=-\left(a e^{2}+f b^{2}-2 b d e\right)
\end{gathered}
$$


For any given geometric parameters and orientation parameters, generally, $D \neq 0$ and $\delta<0$ for general values of $X_{V}$, so Eq. (76) indicates a set of hyperbolas shown in Fig. 19. Geometric parameters and orientation parameters used in these examples are given as $R_{a}=2, R_{b}=1.5$, $\beta_{0}=\Pi / 2,(\phi, \theta, \psi)=(\Pi / 3, \Pi / 6,0)$.

Further research shows that for any given geometric parameters, $R_{a}, R_{b}$ and $\beta_{0}$, and orientation parameters, $(\phi, \theta, \psi)$, of the manipulator, $D$ is a quartic expression while $\delta$ is a quadratic expression with respect to the single variable $X_{V}$. Generally, there are four real roots when $D=0$ and $\delta \neq 0$, and, in each of the four cases, Eq. (76) degenerates into two intersecting lines. For the same reason, there is one real root of multiplicity two when $\delta=0$ and $D=0$; in this case, Eq. (83) degenerates into a parabola. In order to demonstrate the aforementioned theoretical results, a 6/6-GSP will be studied, whose geometric parameters and the orientation parameters are given as follows: $R_{a}=2, R_{b}=1.5, \beta_{0}=\Pi / 2,(\phi, \theta, \psi)=(\Pi / 3$, $\Pi / 6,0)$. Please note that the following calculations are all based on these parameters.

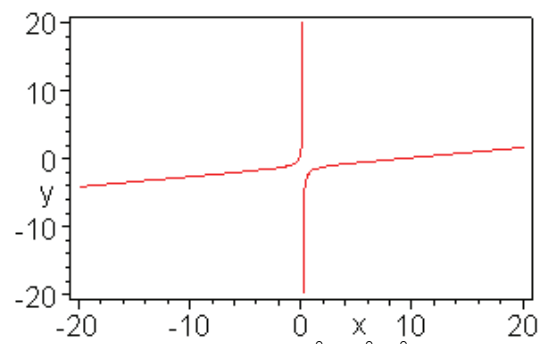

(a) for orientation $\left(60^{\circ}, 30^{\circ}, 0^{\circ}\right), X_{V}=0$

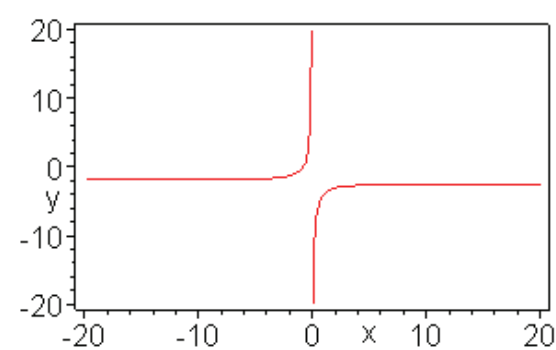

(b) for orientation $\left(60^{\circ}, 30^{\circ}, 0^{\circ}\right), X_{V}=-1$

Fig. 17. Singularity loci in parallel principal-planes for general values of $X_{V}$

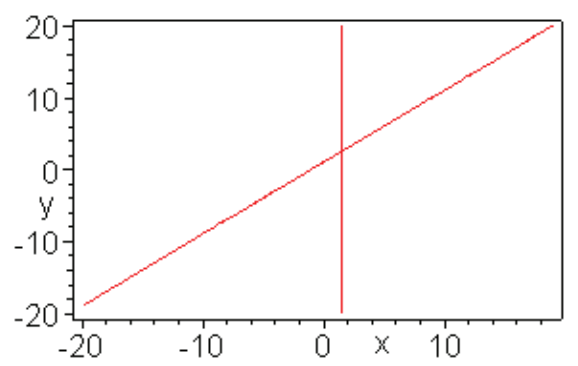

Fig. 18. The first case of two intersecting lines

\subsubsection{First Case of Two Intersecting Lines}

The intersecting line UV passes through point $C_{3}$ and then point $V$ coincides with point $C_{3}$. When $X_{V 1}=(\sqrt{6}+\sqrt{2}) / 2$, Eq. (76) degenerates into two intersecting lines, as shown in Fig. 18.

$$
(8 x-3(\sqrt{6}+\sqrt{2}))\left(x+k_{1} y+c_{1}\right)=0
$$

\subsubsection{Second Case of Two Intersecting Lines}

The intersecting line UV passes through point $C_{4}$ and then point $V$ coincides with point $C_{4}$. When 


$$
X_{V 2}=(\sqrt{6}-\sqrt{2}) / 2
$$

Eq. (76) also degenerates into two intersecting lines

$$
(8 x-3(\sqrt{6}-\sqrt{2}))\left(x+k_{2} y+c_{2}\right)=0
$$

The first part of Eq. (81) is

$$
8 x-3(\sqrt{6}-\sqrt{2})=0
$$

which is a line parallel to $y$-axis. Meanwhile, it can be proved that point $B_{1}$ is located on the base plane. Similarly, when $B_{1}$ coincides with $C_{4}$, the singularity of this point is of the first special-linear-complex singularity. Similarly, when $B_{1}$ does not coincide with $C_{4}$, singularities corresponding to points lying in line of Eq. (82) are of the general-linearcomplex singularity.

The second part of Eq. (81) denotes another line. Singularities corresponding to points lying in this line are all of the general-linear-complex singularity.

\subsubsection{Third Case of Two Intersecting Lines}

When $X_{V 3}=3(\sqrt{6}+\sqrt{2}) / 2, D=0$ and $\delta \neq 0$, Eq. (76) degenerates into two intersecting lines

$$
(8 x+(9 \sqrt{2}+3 \sqrt{6}))\left(x+k_{3} y+c_{3}\right)=0
$$

\subsubsection{The Fourth Case of Two Intersecting Lines}

When $X_{V 4}=(11 \sqrt{6}-3 \sqrt{2}) / 12, D=0$ and $\delta \neq 0$, Eq. (76) degenerates into two intersecting lines

$$
(24 x-(15 \sqrt{2}+5 \sqrt{6}))\left(x+k_{4} y+c_{4}\right)=0
$$

The first part of Eq. (84) is

$$
24 x-(15 \sqrt{2}+5 \sqrt{6})=0
$$

which is a line parallel to $y$-axis of frame $\mathbf{V}$ - $x y$. In particular, there are three special points at which all the segments associated with the six extensible links of the manipulator intersect one common line, respectively

\subsubsection{One Case of a Parabola}

When $X_{V 5}=(7 \sqrt{6}+3 \sqrt{2}) / 6, \delta=0$ and $D \neq 0$, Eq. (76) degenerates into a parabola, as shown in Fig. 23

$$
(528 \sqrt{6}-912 \sqrt{2}) x^{2}+(1423 \sqrt{3}-2472) x+(504-288 \sqrt{3}) y+513 \sqrt{6}-909 \sqrt{2}=0
$$

The manipulator is always singular corresponding to points lying in the parabola. Similarly, there are three special points at which all the segments associated with the six extensible links of the manipulator intersect one common line.

Based on the analyses described above, it can be concluded that the singularity loci of this class of the 6/6-Gough-Stewart manipulators in parallel principal-sections are always 
quadratic expressions that generally include infinite hyperbolas. However, for four parallel locations of the principal-section, the quadratic expression degenerates into two intersecting lines respectively, and in one location the quadratic expression is a parabola when $\theta \neq 0$.

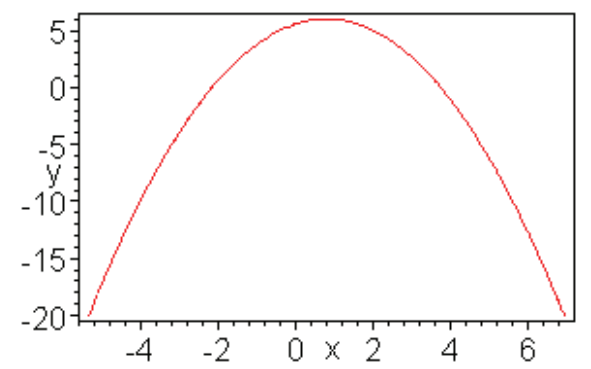

Fig. 19. One case of a parabola

\subsubsection{Singularity analysis when $\theta=0$}

When $\theta=0$, the moving platform is parallel to the base one. Meanwhile, Eq. (94) can be reduces as follows

$$
Z^{3} \cos (\phi+\psi)=0
$$

1. When $Z=0$, the moving and the base platforms are coincident. In this special configuration, the manipulator has three DOFs: two rotational freedoms and one translational freedom.

2. When $(\phi+\psi)= \pm 90^{\circ}$, for the 6/6-Gough-Stewart manipulator, it is the singularity proposed by Fichter (1986).

As we have discussed, the singularity loci of this class of the 6/6-Gough-Stewart manipulators in parallel principal-sections include infinite hyperbolas, four cases of two intersecting lines, and one case of a parabola when $\theta=0$.

From analytic geometry, there are five different types of quadric surface with hyperbola sections: hyperbolic cylinder, hyperbolic paraboloid, hyperboloid of one sheet, hyperboloid of two sheets, and conic surface. However, none of these can contain infinite hyperbolas, one case of a parabola, and four cases of two intersecting lines simultaneously. Therefore, the singularity locus equation of this class of 6/6-Gough-Stewart manipulators considered in three-dimensional space is a special irresolvable polynomial expression of degree three, whose cross-sections in parallel principal-sections contain one case of a parabola, four cases of two intersecting lines and infinite hyperbolas.

\section{Conslusion}

6.1 A necessary and sufficient condition that the three velocities of three non-collinear points in a rigid body can reflect that the body has a possible twist motion is that the intersecting point of three normal planes of three velocities lies in the plane determined by the three points. This is also the necessary and sufficient condition of occuring singularity for a parallel mechanism, when all actuators are locked.

6.2 Based on the singularity kinematics principle and Singularity-Equivalent-Mechanism method, the structure and property of the singularity surface of 3/6-Gough-Stewart platform for all different orientations $(\phi, \theta, \psi)$ can be finally concluded as follows 
6.2.1 When $\theta=0$, the mobile platform and the base one are parallel with each other. The special configuration of the mechanism is $\left(90^{\circ}, 0,0\right)$ which is proposed by Fichter (1986). The mechanism is singular whatever the position of the mobile platform is. It belongs to the general-linear-complex singularity.

6.2.2 When $\theta=0, Z=0$, the mobile platform and the base one are coincident. The mechanism is singular whatever the other two Euler angles are. The mechanism has three DOF: two rotational freedoms and one translational freedom. They belong to the first or the second special-linear-complex singularity, respectively (Huang; Chen; Li 2003).

6.2.3 When $\theta \neq 0, \phi \neq \pm 30^{\circ}, \pm 90^{\circ}$, and $\pm 150^{\circ}$ and $\psi \neq \pm 30^{\circ}, \pm 90^{\circ}$, and $\pm 150^{\circ}$, the singularity loci for this orientation include a plane (Hunt plane) and a hyperbolic paraboloid (Huang; Chen; Li 2003). The intersecting line UV between the mobile platform and the base one is parallel to some side of the basic triangle $\mathrm{A}_{1} \mathrm{~A}_{3} \mathrm{~A}_{5}$.

6.2.4 When $\theta \neq 0, \phi \neq \pm 30^{\circ}, \pm 90^{\circ}$, and $\pm 150^{\circ}$ and $\psi= \pm 30^{\circ}, \pm 90^{\circ}$, or $\pm 150^{\circ}$, the singularity loci are three intersecting planes (Huang; Chen; Li 2003).

6.2.5 When $\theta \neq 0, \phi \neq \pm 30^{\circ}, \pm 90^{\circ}$, and $\pm 150^{\circ}$ and $\psi= \pm 30^{\circ}, \pm 90^{\circ}$, or $\pm 150^{\circ}$, the singularity loci for this orientation also include a plane and a hyperbolic paraboloid (Huang; Chen; Li 2003).

6.2.6 When $\theta=0$, neither $\phi$ nor $\psi$ is equal to any one of the angles, $\pm 30^{\circ}, \pm 90^{\circ}, \pm 150^{\circ}$. This is the most general case for 3/6-GSP. The singularity equation for this orientation is a special irresolvable polynomial expression of degree three, and the structure of the singularity loci in infinite parallel principal sections includes a parabola, four pairs of intersecting straight lines and infinity of hyperbolas (Huang and Cao 2006).

6.2.7 There is only one instantaneous freedom forming a twist with $h^{m} \neq 0$, appearing when $3 / 6-$ GSP is singular for infinite general orientations. But there are seven special situations in which the instantaneous motion is a pure rotation with $h^{m}=0$. However, under the case $\theta=0$, $Z=0$ the instantaneous motion is a pure translation with $h^{m=\infty}$.

6.2.8 According to the singularity-equivalent-mechanism, the singularity can occur at any point all over the $\theta$-plane. That is to say, the singularity can occur everywhere in the workspace of the mechanism. But the singularity orientation of the platform in different points may be different.

6.2.9 The planar section parallel to the mobile platform is named principal section of the singularity surface. The singularity expressions in infinite parallel principal sections are always quadratic.

6.3 The structure and property of the singularity loci of this class of 6/6-Gough-Stewart manipulators for all different orientations can be finally concluded as follows (Huang and Cao 2005)

6.3.1 The singularity locus equation of degree three, is of a special irresolvable polynomial expression whose cross-sections in parallel principal-sections contain one parabola, four pairs of intersecting lines, and infinite hyperbolas.

6.3.2 The graphical representations of the singularity locus of this class of the 6/6-GoughStewart manipulators are quite complex and various for different orientations. The most complex graphic of the singularity loci looks like a trifoliate surface with two holes.

6.3.3 We find an incredible phenomenon, for this class of the 6/6-Gough-Stewart manipulators, there are also some special singularity cases where six lines associated with the six extensible links of the manipulator can intersect one common line and the remnant motion of the manipulator is a pure rotational motion. Even for the same orientation of the manipulator, there are two or more positions of the manipulator at which the six lines of the manipulator all intersect one common line simultaneously. 
6.3.4 When $\theta=0, Z=0$, the manipulator also has three remnant DOFs: two rotational freedoms and one translational freedom.

\section{Acknowledgment}

The research work reported here is supported by the NSFC under Grant No. 59885006 and 50275129, 50905075 and Supported by the Self-determined Research Program of Jiangnan University under Grant No. JUSRP10908.

\section{References}

Ball, R. S. (1900). Theory of Screw. Cambridge:Cambridge University Press, 1900.

Cao, Y.; Huang, Z. \& Ge, Q. J. (2005). Orientation-Singularity and Orientation Capability Analysis of The Stewart-Gough Manipulator, ASME 2005 paper DETC2005-84556, 2005.

Chan, V. K. \& Ebert-Uphoff, I. (2000). Investigation of the deficiencies of parallel manipulators in singular configurations through the Jacobian nullspace. Proc. IEEE Int. Conf. on Robotics and Automation, Seoul, Korea, 2000.

Collins, C. L. \& Long, G. L. (1995). The Singularity Analysis of an In-Parallel Hand Controller for ForceReflected Teleoperation," IEEE Transactions on Robotics and Automation, Vol. 11, No. 5, October, 1995.

Collins, C. L. \& McCarthy, J. M. (1997). The singularity loci of two triangular parallel manipulator. IEEE ICAR 97', Monterey, CA, July 7:9, pp. 473-478, 1997.

Collins, C. L. \& McCarthy, J. M. (1998). The quaric singularity surfaces of planar platforms in the Clifford algebra of the projective plane, Mechanism and Machine Theory, 33:7, pp.931-944, 1998.

Di Gregorio, R.(2001). Analytic Formulation of the 6-3 Fully-Parallel Manipulator's Singularity Determination," Robotica, 19, pp.663-667, 2001.

Di Gregorio, R. (2002). Singularity-Locus Expression of a Class of Parallel Mechanisms, Robotica, 20, pp. 323$328,2002$.

Di Gregorio, R. (2004). Properties of the SX-YS-ZS Structures and Singularity Determination in Parallel Manipulators which Generate Those Structures. Proceedings of DETC'04: ASME 2004 Design Engineering Technical Conferences and Computers and Information in Engineering Conference September 28 - October 2, 2004, Salt Lake City, Utah USA, 2004.

Ebert-Uphoff, I.; Lee, J.-K. \& Lipkin, H. (2000). Characteristic Tetrahedron of Wrench Singularities for Parallel Manipulators with Three Legs. Proc. of A Symposium Commemorating the Legacy, Works, and Life of Sir Robert Stawell Ball Upon the $100^{\text {th }}$ Anniversary of "A Treatise on the Theory of Screws" University of Cambridge, Trinity College, 2000.

Fichter, E. F. (1986). A stewart platform-based manipulator: General theory and practical construction, Int. J. Rob. Res. 5, pp.157-182, 1986.

Gough, V. E. (1956-57). Contribution to discussion to papers on research in automobile stability and control and in tyre performance. Proc. Auto. Div. Instn mech. Engrs, p.392. by Cornell staff, pp. 392-397, 1956-57.

Gosselin, C. \& Angeles, J. (1990). Singularity analysis of closed-loop kinematic chains, IEEE Trans. Rob. Autom. 6, pp. 281-190, 1990

Hao, F. \& McCarthy, J. M. (1998). Conditions for Line-Based Singularities in Spatial Platform Manipulator, Journal of Robotic Systems, 15(1), pp. 43-55, 1998.

Huang Z. and Qu Y. Y. (1987). The analysis of the special configuration of the spatial parallel manipulators, The $5^{\text {th }}$ National Mechanism Conference, Lu Shan, China, pp. 1-7, 1987.

Huang, Z.; Kong, L.F. \& Fang, Y.F. (1997). Theory and Control of Parallel Robotic Mechanisms Manipulator. Beijing, China. Publisher of Mechanical Industry, 1997.

Huang, Z.; Zhao, Y.; Wang, J. \& Yu, J. J. (1999). Kinematic principle and geometrical Condition of generallinear-complex special configuration of parallel manipulators, Mechanism Machine Theory, 34, pp. 1171$1186,1999$.

Huang, Z.; Chen, L.H. \& Li, Y. W. (2003). The Singularity Principle and Property of Stewart manipulator, Journal of Robotic Systems, 20:4, pp.163-176, 2003.

Huang, Z. \& Cao, Y. (2005). Property Identification of the Singularity Loci of a Class of Gough- Stewart Manipulators, The International Journal of Robotics Research, 24:8, pp. 675-685, 2005 
Huang, Z.; Cao, Y.; Li Y. W.,\& Chen L.H. (2006). Structure and Property of the Singularity Loci of the 3/6Stewart-Gough Platform for General Orientations, Robotica, 2006, 24, pp. 75-84, 2006.

Hunt, K.H.,(1978). Kinematic Geometry of Mechanisms. Oxford, UK: Oxford University Press, 1978.

Hunt, K.H. (1983). Structural kinematics of in-parallel-actuated robot-arms, ASME J. Mech. Transmissions Autom. Design, 105, pp. 705-712, 1983.

Karger, A. \& Husty, M., (1998). Architecture singular parallel manipulators, Proceedings of ARK'98 (J. Lenarcic and M. Husty eds), pp. 445-454, 1998.

Karger, A. (2001). Singularities and self-motions of equiform platforms. Mechanism and Machine Theory, 36:7, pp. 801-805, 2001.

Kong, X W. (1998). Generation of 6-SPS Parallel Manipulators," Proc.of the 1998 ASME Design Engineering Technical Conference,Atlanta, USA, 98DETC/MECH-5952, 1998.

Kong, X. \& Gosselin, C. M., (2001). Uncertainty Singularity Analysis of Parallel Manipulators Based on the Instability Analysis of Structures, The International Journal of Robotics Research, Vol 20 :11, pp. 847-856, 2001.

Kong, X. \& Gosselin, C.M. (2002). Generation of Architecturally Singular 6-SPS Parallel Manipulators with Linearly Related Planar Platforms. Electronic Journal of Computational Kinematics (EJCK), May 2002. 1(1), Paper No. 7, 2002

Ma, O. \& Angeles, J.,(1991). Architecture Singularity of Platform Manipulators, IEEE Int.Conf.on Robotics and Automation. Sacramento, USA, pp. 1542-1547, 1991.

Mayer St-Onge, B. \& Gosselin, C. (2000). Singularity analysis and representation of the general Gough-Stewart platform, Int. J. Rob. Res. Vol.19, No. 3, pp, 271-288, 2000.

McAree, P.R. \& Daniel, R. W.(1999). An Explanation of never-special Assembly Changing Motions for 3-3 Parallel Manipulators, Int. J. of. Robotics Research, 18:6, pp. 556-574, 1999.

Merlet, J. P.( 1988). Parallel manipulator part 2: Singular configurations and grassmann geometry. Technical report, INRIA, Sophia Antipolis, France, 1988.

Merlet J.P. (1989). Singular configurations of parallel manipulators and Grassmann geometry, Int. J. Rob. Res. 8, pp. 45-56, 1989.

Parenti-Castelli, V. \& Innocenti, C., (1990). Direct displacement analysis for some classes of spatial parallel mechanisms, Proc. of the 8th CISM-IFToMM Symp. on Theory and Practice of Robots and Manipulators, Cracow, Poland, pp. 126-133, 1990.

Pernkopf, F. \& Husty, M. L. (2002). Sigularity Analysis of Spatial Stewart-Gough Platforms with Planar Base and Platform. Proceedings of DETC.02 ASME 2002 Design Engineering Technical Conferences and Computer and Information in Engineering Conference, Montreal, Canada, September 29 - October 2, 2002, DETC2002/MECH-34267.

Sefrioui, J. \& Gosselin, C. (1994). Étude et représentation des lieux de singularité des manipulateurs parallèles sphériques à trois degrés de liberté avec actionneurs prismatiques, Mechanism Machine Theory, 29, pp. 559$579,1994$.

Sefrioui, J. \& Gosselin, C. (1995). On the quadratic nature of the singularity curves of planar three-degree-offreedom parallel manipulators, Mechanism Machine Theory, 30, pp. 533-551, 1995.

Sugimoto, K. \& Duffy, J.(1981). Special Configuration of Industrial Robots, Proc. of the 11th ISIR, pp: 309-316, 1981.

Stewart, D. (1965). A platform with six degrees of freedom, Proc. Inst. Mech. Eng, 180, pp. 371-378, 1965.

Wang, G. Z. (1998). Singulrity analysis of a class of the 6-6 Stewart platforms. Proc. of DETC'98 1998 ASME Design Engineering Technical Conference, Atlanta, Georgia, USA, 1998.

Wang, J. \& Gosselin, C.M. (1996). Kinematic analysis and singularity loci of spatial four-degree-of-freedom parallel manipulators. Proc. of the 1996 ASME Design Engineering Technical Conference and Computers in Engineering Conference, California, USA, 1996.

Wang, J. \& Gosselin, C. (1997). Kinematic analysis and singularity representation of spatial five-degree-offreedom parallel mechanisms, J Robotic Syst, 14, pp. 851-869, 1997.

Zlatanov, D., Bonev, I.A. \& Gosselin, C.M., (2002), Constraint singularities of parallel mechanisms, Proceedings of the 2002 IEEE International conference on Robotics and Automation, Washington, DC, USA, pp. 496$502,2002$.

Zlatanov, D.; Fenton, R.G. \& Benhabib, B.(1994). Singularity analysis of mechanisms and robots via a motionspace model of the instantaneous kinematics, Proc. of 1994 IEEE Int. Conf. On Rob. And Auto 2 pp. 980991, 1994.

Zlatanov, D, Fenton, RG \& Benhabib, B. (1995). A Unifying Framework for Classification and Interpretation of Mechanism Singularities. ASME J Mechanical Design, 117, pp. 566-572, 1995. 


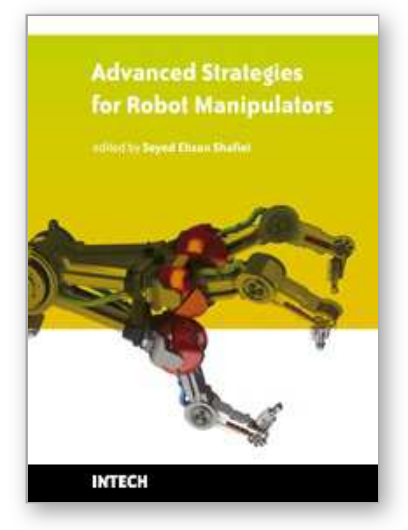

\author{
Advanced Strategies for Robot Manipulators \\ Edited by S. Ehsan Shafiei
}

ISBN 978-953-307-099-5

Hard cover, 428 pages

Publisher Sciyo

Published online 12, August, 2010

Published in print edition August, 2010

Amongst the robotic systems, robot manipulators have proven themselves to be of increasing importance and are widely adopted to substitute for human in repetitive and/or hazardous tasks. Modern manipulators are designed complicatedly and need to do more precise, crucial and critical tasks. So, the simple traditional control methods cannot be efficient, and advanced control strategies with considering special constraints are needed to establish. In spite of the fact that groundbreaking researches have been carried out in this realm until now, there are still many novel aspects which have to be explored.

\title{
How to reference
}

In order to correctly reference this scholarly work, feel free to copy and paste the following:

Yi Cao, Yanwen Li and Zhen Huang (2010). Structure and Property of the Singularity Loci of Gough-Stewart Manipulator, Advanced Strategies for Robot Manipulators, S. Ehsan Shafiei (Ed.), ISBN: 978-953-307-099-5, InTech, Available from: http://www.intechopen.com/books/advanced-strategies-for-robotmanipulators/structure-and-property-of-the-singularity-loci-of-gough-stewart-manipulator

\section{INTECH}

open science | open minds

\section{InTech Europe}

University Campus STeP Ri

Slavka Krautzeka 83/A

51000 Rijeka, Croatia

Phone: +385 (51) 770447

Fax: +385 (51) 686166

www.intechopen.com

\section{InTech China}

Unit 405, Office Block, Hotel Equatorial Shanghai

No.65, Yan An Road (West), Shanghai, 200040, China

中国上海市延安西路65号上海国际贵都大饭店办公楼 405 单元

Phone: +86-21-62489820

Fax: +86-21-62489821 
(C) 2010 The Author(s). Licensee IntechOpen. This chapter is distributed under the terms of the Creative Commons Attribution-NonCommercialShareAlike-3.0 License, which permits use, distribution and reproduction for non-commercial purposes, provided the original is properly cited and derivative works building on this content are distributed under the same license. 